\title{
A Convenient and Efficient Route for the Allylation of Aromatic Amines and $\alpha$ - Aryl Aldehydes with Alkynes in the Presence of a $\mathrm{Pd}(0) / \mathrm{PhCOOH}$ Combined
} Catalyst System

Nitin T. Patil, ${ }^{\mathrm{a}}$ Huanyou Wu, ${ }^{\mathrm{a}}$ Isao Kadota ${ }^{\mathrm{b}}$ and Yoshinori Yamamoto ${ }^{\mathrm{a} *}$

${ }^{a}$ Department of Chemistry, Graduate School of Science, Tohoku University, Sendai 980-8578, Japan

${ }^{b}$ Research and Analytical Center for Giant Molecules, Graduate School of Science, Tohoku University, Sendai 980-8578, Japan

\section{Contents of Supporting Information}

\author{
(42 Pages)
}

Page S-1: Title of the paper, author's name and address along with the contents.

Page S-3: General procedure for the allylation of anilines and characterization data of compounds 6a-c.

Page S-4: Characterization data of compounds $6 \mathbf{f}, \mathbf{6 h}, \mathbf{6 i}, \mathbf{6 n}, \mathbf{6 p}$.

Page S-5: Characterization data of compounds $\mathbf{1 0}$ and references

Page S-6: ${ }^{1} \mathrm{H}$ NMR spectrum of compound 3a

Page S-7: ${ }^{1} \mathrm{H}$ NMR spectrum of compound $\mathbf{3 b}$

Page S-8: ${ }^{1} \mathrm{H}$ NMR spectrum of compound 3c

Page S-9: ${ }^{1} \mathrm{H}$ NMR spectrum of compound 3d

Page S-10: ${ }^{1} \mathrm{H}$ NMR spectrum of compound $\mathbf{3 e}$

Page S-11: ${ }^{1} \mathrm{H}$ NMR spectrum of compound $\mathbf{3 h}$

Page S-12: ${ }^{1} \mathrm{H}$ NMR spectrum of compound $\mathbf{3 i}$

Page S-13: ${ }^{1} \mathrm{H}$ NMR spectrum of compound $\mathbf{6 a}$

Page S-14: ${ }^{1} \mathrm{H}$ NMR spectrum of compound $\mathbf{6 b}$

Page S-15: ${ }^{1} \mathrm{H}$ NMR spectrum of compound $\mathbf{6 c}$

Page S-16: ${ }^{1} \mathrm{H}$ NMR spectrum of compound $\mathbf{6 e}$

Page S-17: ${ }^{1} \mathrm{H}$ NMR spectrum of compound $\mathbf{6 f}$

Page S-18: ${ }^{1} \mathrm{H}$ NMR spectrum of compound $\mathbf{6 g}$

Page S-19: ${ }^{1} \mathrm{H}$ NMR spectrum of compound $\mathbf{6 h}$

Page S-20: ${ }^{1} \mathrm{H}$ NMR spectrum of compound $\mathbf{6 i}$

Page S-21: ${ }^{1} \mathrm{H}$ NMR spectrum of compound $\mathbf{6 j}$

Page S-22: ${ }^{1} \mathrm{H}$ NMR spectrum of compound $\mathbf{6 k}$

Page S-23: ${ }^{1} \mathrm{H}$ NMR spectrum of compound $\mathbf{6 l}$

Page S-24: ${ }^{1}$ H NMR spectrum of compound $\mathbf{6 m}$

Page S-25: ${ }^{1} \mathrm{H}$ NMR spectrum of compound $\mathbf{6 n}$

Page S-26: ${ }^{1} \mathrm{H}$ NMR spectrum of compound $\mathbf{6 0}$

Page S-27: ${ }^{1} \mathrm{H}$ NMR spectrum of compound $\mathbf{6 p}$

Page S-28: ${ }^{1} \mathrm{H}$ NMR spectrum of compound $\mathbf{6 q}$

Page S-29: ${ }^{1} \mathrm{H}$ NMR spectrum of compound $\mathbf{1 0}$ 
Page S-30: General procedure for the $\alpha$-allylation of aldehydes and characterization data of compounds $12 a, 12 b$ and $12 c$.

Page S-31: Characterization data of compounds 12d, 12e, 12f, $12 \mathrm{~g}$ and $\mathbf{1 2 h}$.

Page S-32: Characterization data of compounds $\mathbf{1 2 \mathbf { i }}, \mathbf{1 2 \mathbf { j }}$ and references

Page S-33: ${ }^{1} \mathrm{H}$ NMR spectrum of compound 12a

Page S-34: ${ }^{1} \mathrm{H}$ NMR spectrum of compound 12b

Page S-35: ${ }^{1} \mathrm{H}$ NMR spectrum of compound 12c

Page S-36: ${ }^{1} \mathrm{H}$ NMR spectrum of compound 12d

Page S-37: ${ }^{1} \mathrm{H}$ NMR spectrum of compound 12e

Page S-38: ${ }^{1} \mathrm{H}$ NMR spectrum of compound $\mathbf{1 2 f}$

Page S-39: ${ }^{1} \mathrm{H}$ NMR spectrum of compound $\mathbf{1 2 g}$

Page S-40: ${ }^{1}$ H NMR spectrum of compound $\mathbf{1 2 h}$

Page S-41: ${ }^{1} \mathrm{H}$ NMR spectrum of compound $\mathbf{1 2 i}$

Page S-42: ${ }^{1} \mathrm{H}$ NMR spectrum of compound $\mathbf{1 2} \mathbf{j}$ 
General Procedure for the allylation of aromatic amines. The reaction of $N$-methylaniline 1a with 1-phenyl-1-propyne $\mathbf{2 a}$ is representative. To a mixture of $N$-methylaniline $1 \mathbf{a}(0.092 \mathrm{~g}, 0.859$ mmol), 1-phenyl-1-propyne 2a (0.100 g, $0.859 \mathrm{mmol})$, tetrakis(triphenylphosphine)palladium $(0.050 \mathrm{~g}, 0.043 \mathrm{mmol})$ in dry 1,4-dioxane $(2 \mathrm{~mL})$ was added benzoic acid $(0.010 \mathrm{~g}, 0.086 \mathrm{mmol})$, and the mixture was stirred at $100{ }^{\circ} \mathrm{C}$ for $8 \mathrm{~h}$. The reaction mixture was then filtered through a short silica gel column using ether as an eluent, and the filtrate was concentrated. The residue was purified by a silica gel column chromatography (hexane/AcOEt, 9:1) to give the allylated product 3a $(0.194 \mathrm{~g}, 93 \%)$ as an oil.

Structure of $3 \mathbf{a},{ }^{1} \mathbf{3 b},{ }^{2} \mathbf{3 c},{ }^{3} \mathbf{3 d},{ }^{4} \mathbf{3 e},{ }^{4} 3 \mathbf{h},{ }^{5} 3 \mathbf{3 i},{ }^{5} \mathbf{6 e},{ }^{6} \mathbf{6 g},{ }^{7} \mathbf{6 j},{ }^{8} \mathbf{6 k},{ }^{9} 6 \mathbf{6},{ }^{10} \mathbf{6 m},{ }^{9} 6 \mathbf{6},{ }^{9} 6 \mathbf{q}^{11}$ are known in the literature. The characterization data for the newly synthesized compounds $6 \mathbf{a}, \mathbf{6 b}, \mathbf{6 c}, \mathbf{6 f}, \mathbf{6 h}, \mathbf{6 i}, \mathbf{6 n}$, 6p, 10 as well as the copies of the ${ }^{1} \mathrm{H}$ NMR spectra of all monoallylation products are given below.

N-Cinnamyl-2,4-dimethoxybenzenamine (6a). IR (neat) $3412 \mathrm{~cm}^{-1},{ }^{1} \mathrm{H} \mathrm{NMR}\left(\mathrm{CDCl}_{3}, 400 \mathrm{MHz}\right)$ $\delta$ 7.38-7.18 (m, 5H), $6.60(\mathrm{~d}, J=15.2 \mathrm{~Hz}, 1 \mathrm{H}), 6.57(\mathrm{~d}, J=8.4 \mathrm{~Hz}, 1 \mathrm{H}), 6.45(\mathrm{~d}, J=2.8 \mathrm{~Hz}, 1 \mathrm{H})$, $6.40(\mathrm{~d}, J=2.8 \mathrm{~Hz}, 1 \mathrm{H}), 6.35(\mathrm{dt}, J=15.2,5.6 \mathrm{~Hz}, 1 \mathrm{H}), 3.89(\mathrm{~d}, J=5.6 \mathrm{~Hz}, 2 \mathrm{H}), 3.82(\mathrm{~s}, 3 \mathrm{H}), 3.74$ $(\mathrm{s}, 3 \mathrm{H}) ;{ }^{13} \mathrm{C} \mathrm{NMR}\left(\mathrm{CDCl}_{3}, 100 \mathrm{MHz}\right) \delta 151.9,147.8,136.8,132.1,131.0,128.6,127.9,127.4$, 126.1, 110.5, 103.6, 99.0, 55.6, 55.3, 46.6; HRMS calcd for $\mathrm{C}_{17} \mathrm{H}_{19} \mathrm{NO}_{2}\left(\mathrm{M}^{+}\right)$269.1416, found 269.1410 .

$\boldsymbol{N}$-(4-chlorocinnamyl)-2,4-Dimethoxybenzenamine (6b). IR (neat) $3406 \mathrm{~cm}^{-1},{ }^{1} \mathrm{H} \mathrm{NMR}\left(\mathrm{CDCl}_{3}\right.$, $400 \mathrm{MHz}) \delta 7.31-7.21(\mathrm{~m}, 4 \mathrm{H}), 6.58(\mathrm{~d}, J=8.8 \mathrm{~Hz}, 1 \mathrm{H}), 6.55(\mathrm{~d}, J=15.6 \mathrm{~Hz}, 1 \mathrm{H}), 6.45(\mathrm{~d}, J=2.4$ $\mathrm{Hz}, 1 \mathrm{H}), 6.38(\mathrm{dd}, J=8.8,2.4 \mathrm{~Hz}, 1 \mathrm{H}), 6.31(\mathrm{dt}, J=15.6,6.0 \mathrm{~Hz}, 1 \mathrm{H}), 3.88(\mathrm{dd}, J=6.0,1.2 \mathrm{~Hz}$, 2H), $3.82(\mathrm{~s}, 3 \mathrm{H}), 3.74(\mathrm{~s}, 3 \mathrm{H}) ;{ }^{13} \mathrm{C} \mathrm{NMR}\left(\mathrm{CDCl}_{3}, 100 \mathrm{MHz}\right) \delta 151.9,147.9,135.4,132.8,132.0$, 129.8, 128.5, 128.2, 127.4, 110.5, 103.7, 99.1, 55.7, 55.4, 46.6; HRMS calcd for $\mathrm{C}_{17} \mathrm{H}_{18} \mathrm{ClNO}_{2}\left(\mathrm{M}^{+}\right)$ 303.1026, found 303.1025.

\section{$N$-(4-methoxycinnamyl)-2,4-Dimethoxybenzenamine (6c).}

IR (neat) $3455 \mathrm{~cm}^{-1},{ }^{1} \mathrm{H} \mathrm{NMR}\left(\mathrm{CDCl}_{3}, 400 \mathrm{MHz}\right) \delta 7.30(\mathrm{~d}, J=8.4 \mathrm{~Hz}, 2 \mathrm{H}), 6.82(\mathrm{~d}, J=8.4 \mathrm{~Hz}$, 2H), $6.59(\mathrm{~d}, J=8.4 \mathrm{~Hz}, 1 \mathrm{H}), 6.54(\mathrm{~d}, J=16.0 \mathrm{~Hz}, 1 \mathrm{H}), 6.44(\mathrm{~d}, J=2.4 \mathrm{~Hz}, 1 \mathrm{H}), 6.38(\mathrm{dd}, J=8.4$, $2.4 \mathrm{~Hz}, 1 \mathrm{H}), 6.21(\mathrm{dt}, J=16.0,6.0 \mathrm{~Hz}, 1 \mathrm{H}), 3.86(\mathrm{dd}, J=6.0,1.2 \mathrm{~Hz}, 2 \mathrm{H}), 3.82$ (s, $3 \mathrm{H}), 3.80$ (s, 3H), $3.74(\mathrm{~s}, 3 \mathrm{H}) ;{ }^{13} \mathrm{C} \mathrm{NMR}\left(\mathrm{CDCl}_{3}, 100 \mathrm{MHz}\right) \delta 158.9,151.9,147.9,133.1,132.1,130.7,129.9$, 
129.6, 128.2, 127.3, 125.1, 113.8, 110.8, 103.7, 99.0, 55.7, 55.4, 55.2, 46.9; HRMS calcd for $\mathrm{C}_{18} \mathrm{H}_{21} \mathrm{NO}_{3}\left(\mathrm{M}^{+}\right)$299.1521, found 299.1525 .

$\mathrm{N}$-Cinnamyl-2,3-dimethylbenzenamine (6f). IR (neat) $3442 \mathrm{~cm}^{-1},{ }^{1} \mathrm{H}$ NMR $\left(\mathrm{CDCl}_{3}, 400 \mathrm{MHz}\right)$ $\delta$ 7.39-7.16 (m, 5H), 7.01 (broad triplet, $J=8.0 \mathrm{~Hz}, 1 \mathrm{H}), 6.65-6.58(\mathrm{~m}, 2 \mathrm{H}), 6.57(\mathrm{~d}, J=8.4 \mathrm{~Hz}$, 1H), 6.36 (ddd, $J=16.0,5.6,2.0 \mathrm{~Hz}, 1 \mathrm{H}$ ), 3.95 (d, $J=5.6 \mathrm{~Hz}, 2 \mathrm{H}$ ), 3.68 (bs, 1H), 2.28 (s, 3H), 2.07 $(\mathrm{s}, 3 \mathrm{H}) ;{ }^{13} \mathrm{C} \mathrm{NMR}\left(\mathrm{CDCl}_{3}, 100 \mathrm{MHz}\right) \delta 145.8,136.8,136.4,131.4,128.5,127.4,127.2,126.1$, 120.3, 119.5, 108.3, 46.5, 20.7, 12.6; HRMS calcd for $\mathrm{C}_{17} \mathrm{H}_{19} \mathrm{~N}\left(\mathrm{M}^{+}\right)$237.1517, found 237.1512.

$\boldsymbol{N}$-Cinnamyl-2-cyanobenzenamine (6h). IR (neat) $3448 \mathrm{~cm}^{-1},{ }^{1} \mathrm{H}$ NMR $\left(\mathrm{CDCl}_{3}, 400 \mathrm{MHz}\right) \delta 7.41$ $7.20(\mathrm{~m}, 7 \mathrm{H}), 6.70(\mathrm{~d}, J=8.4 \mathrm{~Hz}, 1 \mathrm{H}), 6.68(\mathrm{t}, J=7.6 \mathrm{~Hz}, 1 \mathrm{H}), 6.60(\mathrm{~d}, J=16.0 \mathrm{~Hz}, 1 \mathrm{H}), 6.24(\mathrm{dt}, J$ $=16.0,5.6 \mathrm{~Hz}, 1 \mathrm{H}), 4.80(\mathrm{bs}, 1 \mathrm{H}), 4.01(\mathrm{dd}, J=5.6,1.2 \mathrm{~Hz}, 2 \mathrm{H}) ;{ }^{13} \mathrm{C} \mathrm{NMR}\left(\mathrm{CDCl}_{3}, 100 \mathrm{MHz}\right) \delta$ $149.8,136.2,134.0,132.5,131.9,128.4,127.6,126.2,126.1,125.1,117.7,116.5,110.8,95.7,45.2$; HRMS calcd for $\mathrm{C}_{16} \mathrm{H}_{14} \mathrm{~N}_{2}\left(\mathrm{M}^{+}\right)$234.1157, found 234.1159.

$N$-Cinnamyl-2-methoxy-4-nitrobenzenamine (6i). IR (neat) $3413 \mathrm{~cm}^{-1},{ }^{1} \mathrm{H}$ NMR $\left(\mathrm{CDCl}_{3}, 400\right.$ MHz) $\delta 7.88(\mathrm{dd}, J=8.8,2.4 \mathrm{~Hz}, 1 \mathrm{H}), 7.62(\mathrm{~d}, J=2.4 \mathrm{~Hz}, 1 \mathrm{H}), 7.36-7.21(\mathrm{~m}, 5 \mathrm{H}), 6.60(\mathrm{~d}, J=16.0$ $\mathrm{Hz}, 1 \mathrm{H}), 6.54(\mathrm{~d}, J=8.8 \mathrm{~Hz}, 1 \mathrm{H}), 6.25$ (dt, $J=16.0,5.6 \mathrm{~Hz}, 1 \mathrm{H}), 5.23$ (bt, 1H), 4.04 (dt, $J=5.6,1.6$ $\mathrm{Hz}, 2 \mathrm{H}), 3.93$ (s, 3H); ${ }^{13} \mathrm{C} \mathrm{NMR}\left(\mathrm{CDCl}_{3}, 100 \mathrm{MHz}\right) \delta 144.9,143.8,136.9,136.1,132.2,128.4$, 127.6, 126.2, 124.7, 119.6, 106.8, 104.5, 55.7, 44.9; HRMS calcd for $\mathrm{C}_{16} \mathrm{H}_{16} \mathrm{~N}_{2} \mathrm{O}_{3}\left(\mathrm{M}^{+}\right)$284.1161, found 284.1155 .

$N$-Cinnamyl-3-acetylbenzenamine (6n). IR (neat) $3421,1698 \mathrm{~cm}^{-1},{ }^{1} \mathrm{H}$ NMR $\left(\mathrm{CDCl}_{3}, 400 \mathrm{MHz}\right)$ $\delta$ 7.36-7.18 (m, 8H), $6.85(\mathrm{~d}, J=7.6 \mathrm{~Hz}, 1 \mathrm{H}), 6.61(\mathrm{~d}, J=16.0 \mathrm{~Hz}, 1 \mathrm{H}), 6.28(\mathrm{dt}, J=16.0,5.6 \mathrm{~Hz}$, $1 \mathrm{H}), 3.97(\mathrm{~d}, J=5.6 \mathrm{~Hz}, 2 \mathrm{H}), 2.55(\mathrm{~s}, 3 \mathrm{H}) ;{ }^{13} \mathrm{C} \mathrm{NMR}\left(\mathrm{CDCl}_{3}, 100 \mathrm{MHz}\right) \delta 198.5,147.9,137.9$, 136.5, 133.2, 131.5, 129.1, 12.6, 126.2, 126.1, 117.7, 117.5, 111.7, 45.9, 26.6; HRMS calcd for $\mathrm{C}_{17} \mathrm{H}_{17} \mathrm{NO}\left(\mathrm{M}^{+}\right)$251.1310, found 251. 1315 .

$\mathrm{N}$-Cinnamyl-4-phenylbenzenamine (6p). IR (neat) $3434 \mathrm{~cm}^{-1},{ }^{1} \mathrm{H}$ NMR $\left(\mathrm{CDCl}_{3}, 400 \mathrm{MHz}\right)$ $\delta$ 7.45-7.11 (m, 12H), $6.78(\mathrm{~d}, J=7.6 \mathrm{~Hz}, 1 \mathrm{H}), 6.75(\mathrm{~d}, J=7.6 \mathrm{~Hz}, 1 \mathrm{H}), 6.54(\mathrm{~d}, J=15.6 \mathrm{~Hz}, 1 \mathrm{H})$, $6.25(\mathrm{dt}, J=15.6,5.6 \mathrm{~Hz}, 1 \mathrm{H}), 4.15(\mathrm{bs}, 1 \mathrm{H}), 3.90(\mathrm{bs}, 2 \mathrm{H}) ;{ }^{13} \mathrm{C} \mathrm{NMR}\left(\mathrm{CDCl}_{3}, 100 \mathrm{MHz}\right) \delta 144.7$, 
139.3, 136.7, 131.0, 130.0, 129.2, 128.5, 128.4, 127.6, 127.3, 127.0, 126.6, 126.2, 117.0, 110.7, 46.1; HRMS calcd for $\mathrm{C}_{21} \mathrm{H}_{19} \mathrm{~N}\left(\mathrm{M}^{+}\right)$285.1517, found 285.1512.

\section{2-(cinnamylamino)Phenol (10).}

IR (neat) 3426-3450 (br) $\mathrm{cm}^{-1},{ }^{1} \mathrm{H} \mathrm{NMR}\left(\mathrm{CDCl}_{3}, 400 \mathrm{MHz}\right) \delta$ 7.55-7.14 (m, 5H), $6.79(\mathrm{~d}, J=7.2$ $\mathrm{Hz}, 1 \mathrm{H}), 6.70(\mathrm{t}, J=8.8 \mathrm{~Hz}, 2 \mathrm{H}), 6.61(\mathrm{~d}, J=7.6 \mathrm{~Hz}, 1 \mathrm{H}), 6.55(\mathrm{~d}, J=16.0 \mathrm{~Hz}, 1 \mathrm{H}), 6.29$ (dt, $J=$ 16.0, 6.0 Hz, 1H), $3.87(\mathrm{bd}, J=5.2 \mathrm{~Hz}, 2 \mathrm{H}) ;{ }^{13} \mathrm{C} \mathrm{NMR}\left(\mathrm{CDCl}_{3}, 100 \mathrm{MHz}\right) \delta 171.6,144.0,136.7$, 136.2, 133.5, 131.8, 128.7, 128.5, 127.4, 126.0, 121.5, 118.7, 114.8, 113.5, 46.9; HRMS calcd for $\mathrm{C}_{15} \mathrm{H}_{15} \mathrm{NO}\left(\mathrm{M}^{+}\right) 225.1154$, found 225.1156 .

\section{References of Supporting Information:}

1. Kimura, M.; Futamata, M.; Shibata, K.; Tamaru, Y. Chem. Commun., 2003, 234-235.

2. Jin, M.; Zhang, D.; Yang, L.; Liu Y.; Liu, Z. Tetrahedron Letters 2000, 41, 7357-7360.

3. Ozawa, F.; Okamoto, H.; Kawagishi, S.; Yamamoto, S.; Minami, T.; Yoshifuji, M. J. Am. Chem. Soc. 2002, 124, 10968-10969.

4. Barluenga, J.; Trincado, M.; Rubio, E. Gonzalez, J. M. J. Am. Chem. Soc. 2004, 126, 3416-3417.

5. Al-Masum, M.; Meguro, M.; Yamamoto, Y. Tetrahedron Lett. 1997, 38, 6071-6074.

6. Vizgert, R. V.; Sendega, R. V.; Zhovnirchuk, V. M. Zhurnal Organicheskoi Khimii 1969, 5, 926.

7. Yang, S-C.; Tsai, Y-C.; Shue, Y-J. Organometallics 2001, 20, 5326-5330.

8. Arai, Y.; Toda, M. Tsumoru, M. Jpn Kokai Tokkyo Koho, 1985, 23.

9. Moreno-Manas, M.; Morral, L.; Pleixats, R. J. Org. Chem. 1998, 63, 6160-6166.

10. Itoh, T.; Nagata, K.; Kurihara, A.; Miyazaki, M.; Ohsawa A. Tetrahedron Letters 2002, 43, 3105-3108.

11. Shue, Y-J.; Yang, S-C.; Lai, H-C. Tetrahedron Letters 2003, 44, 1481-1485. 


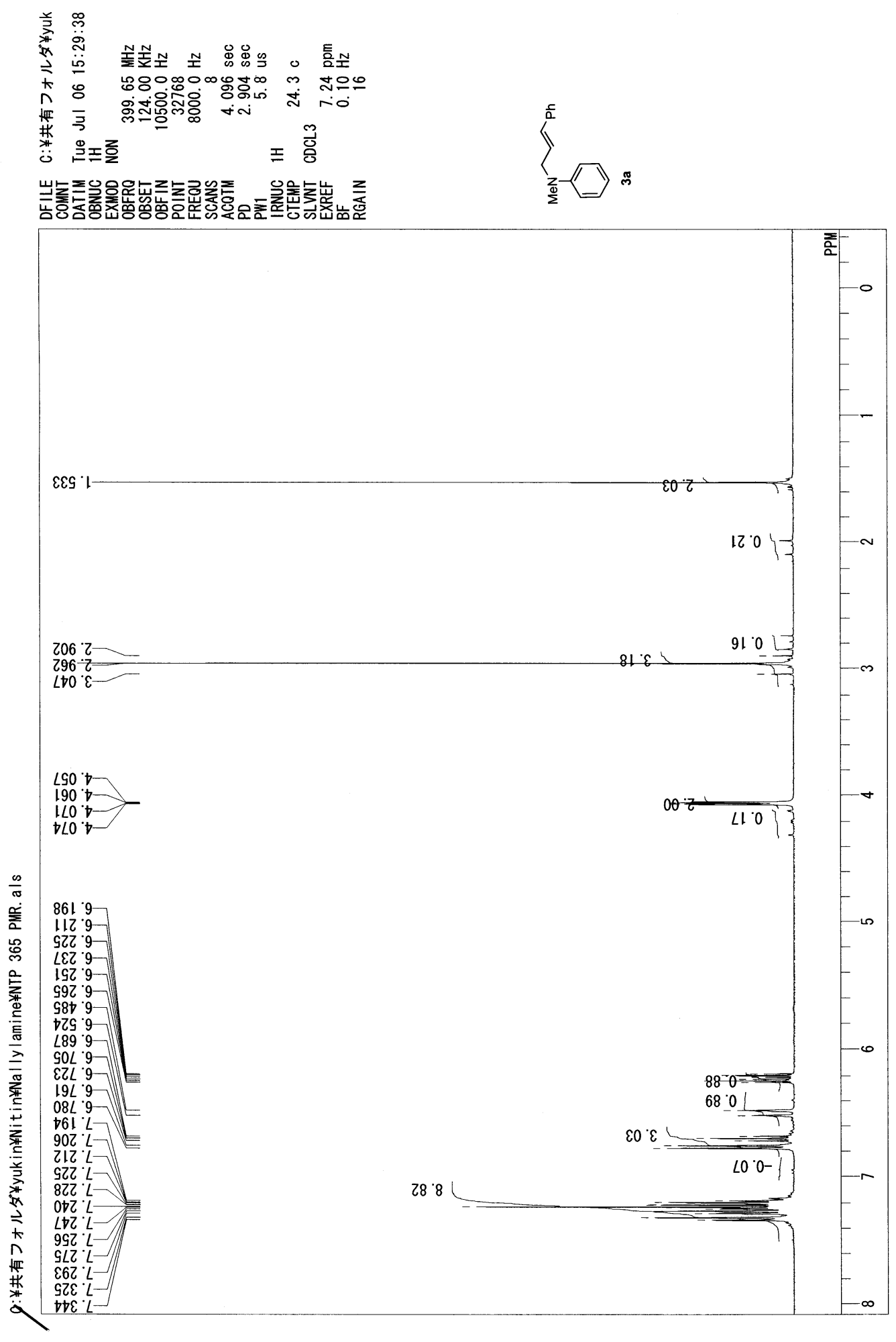




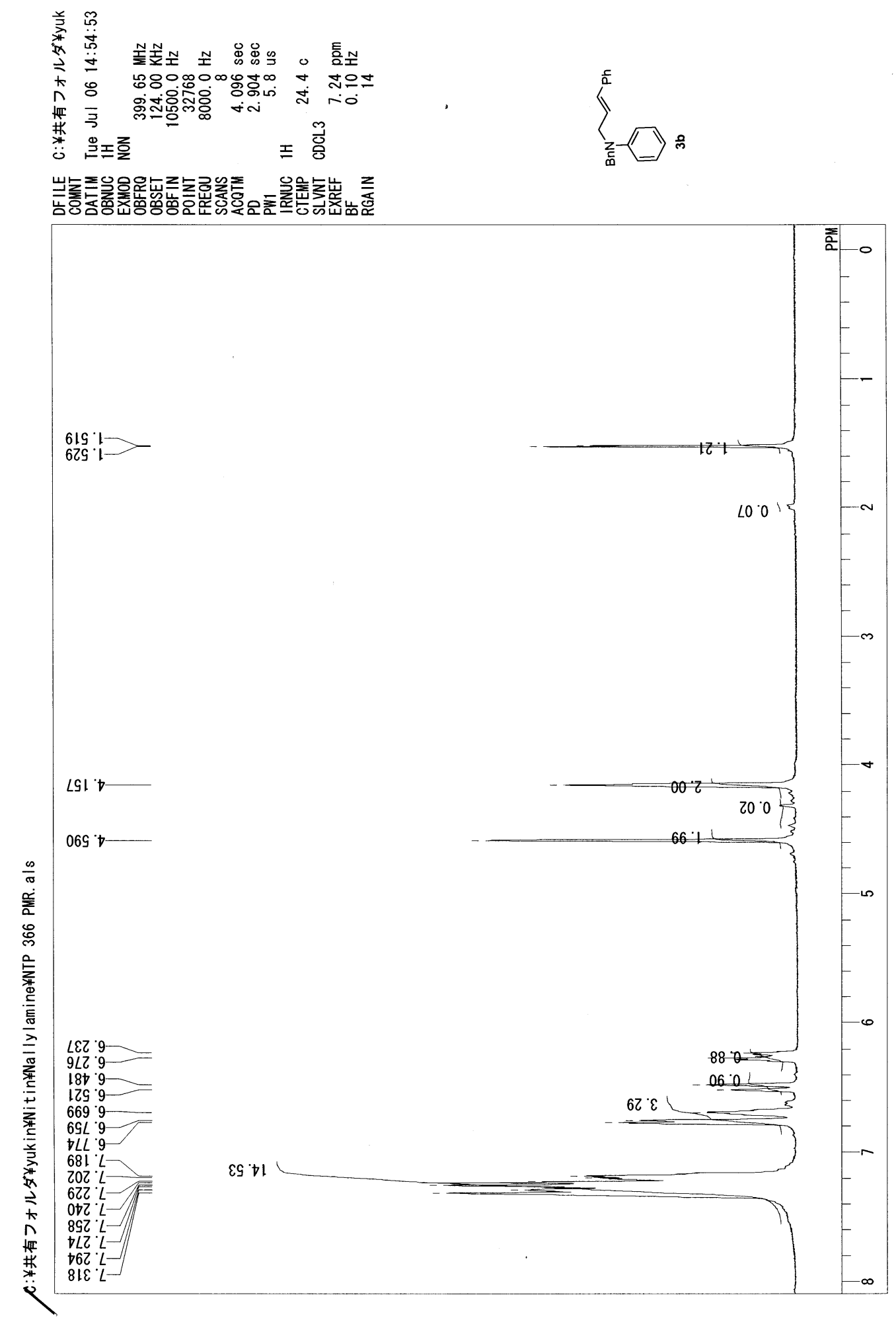



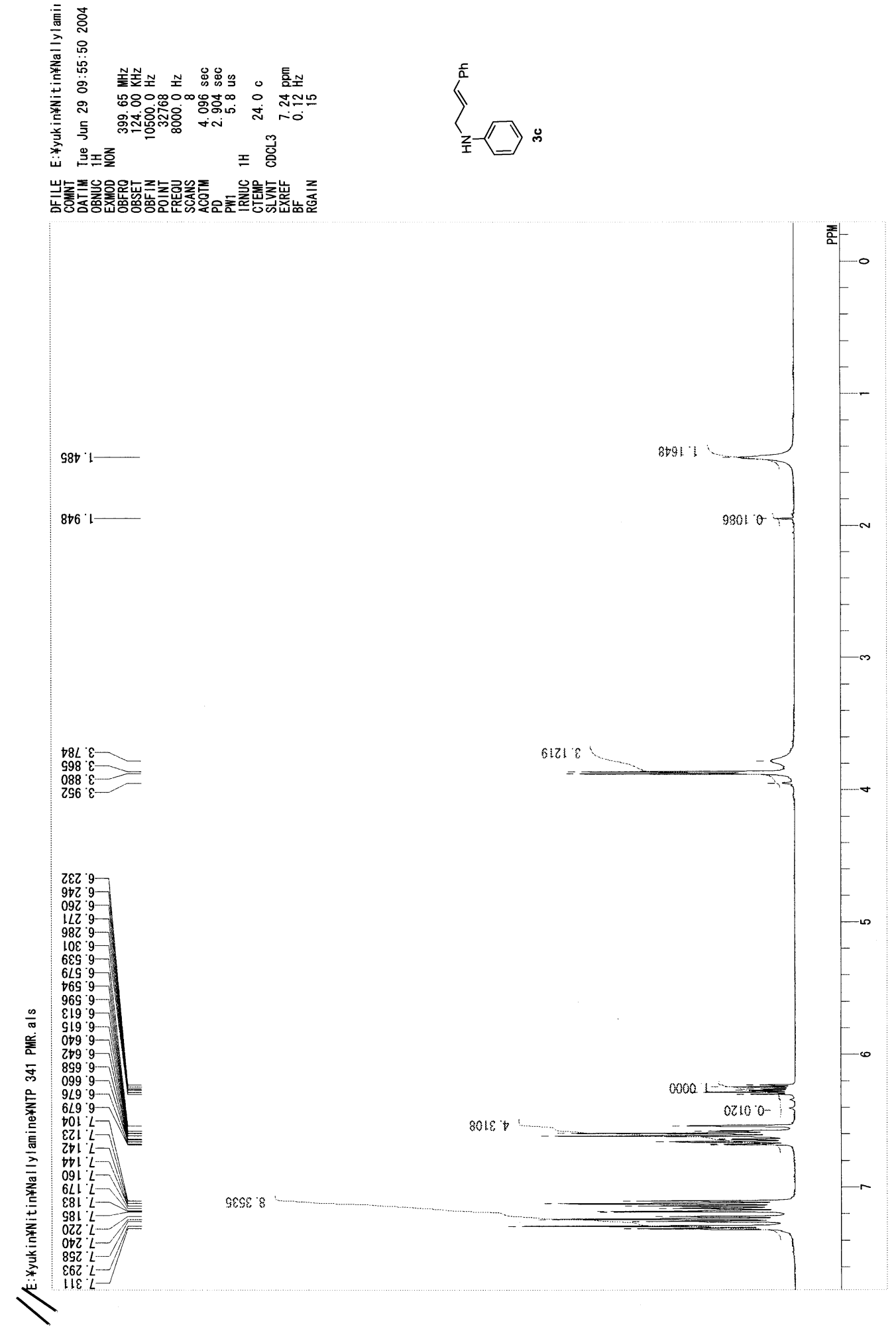


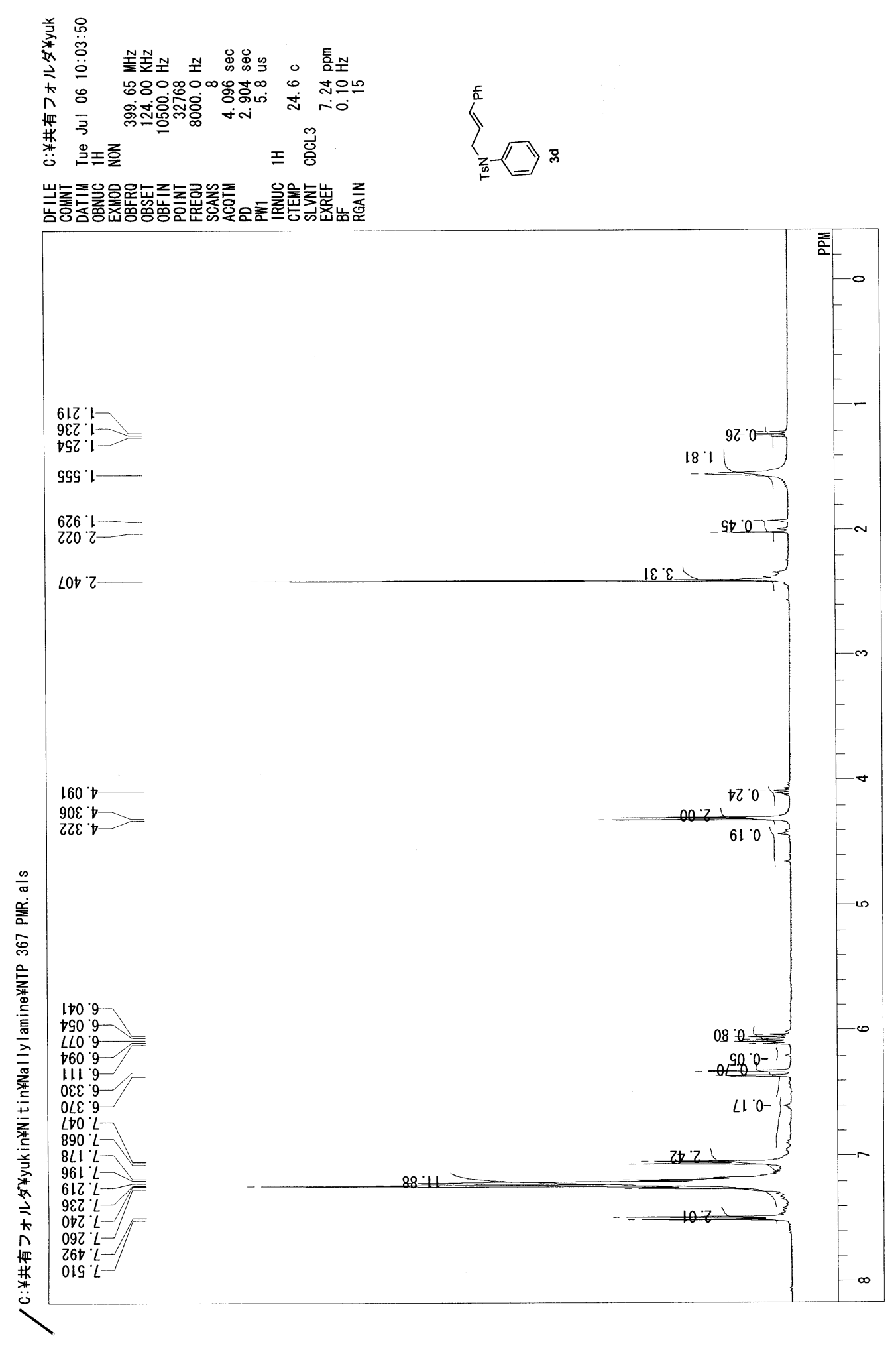




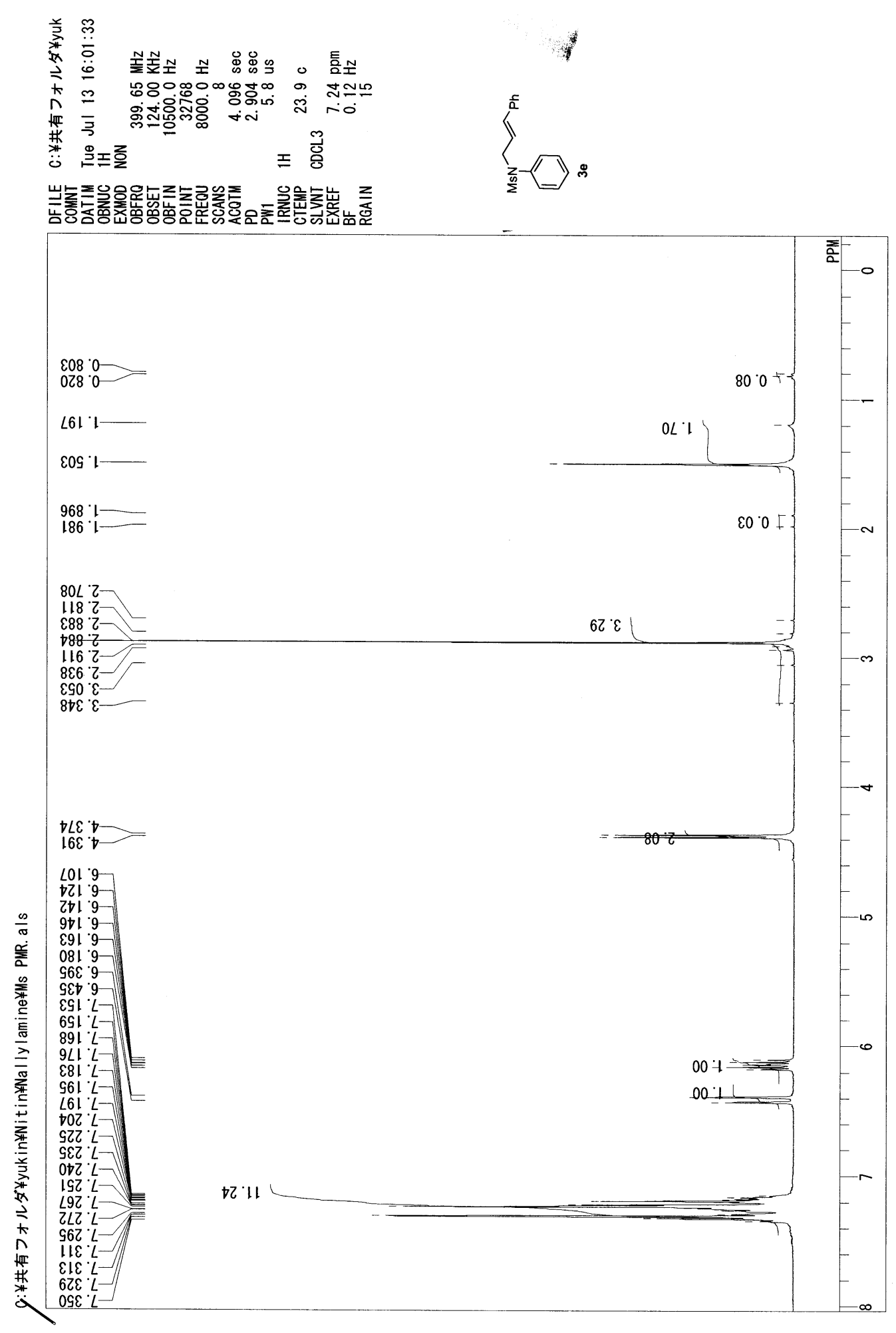




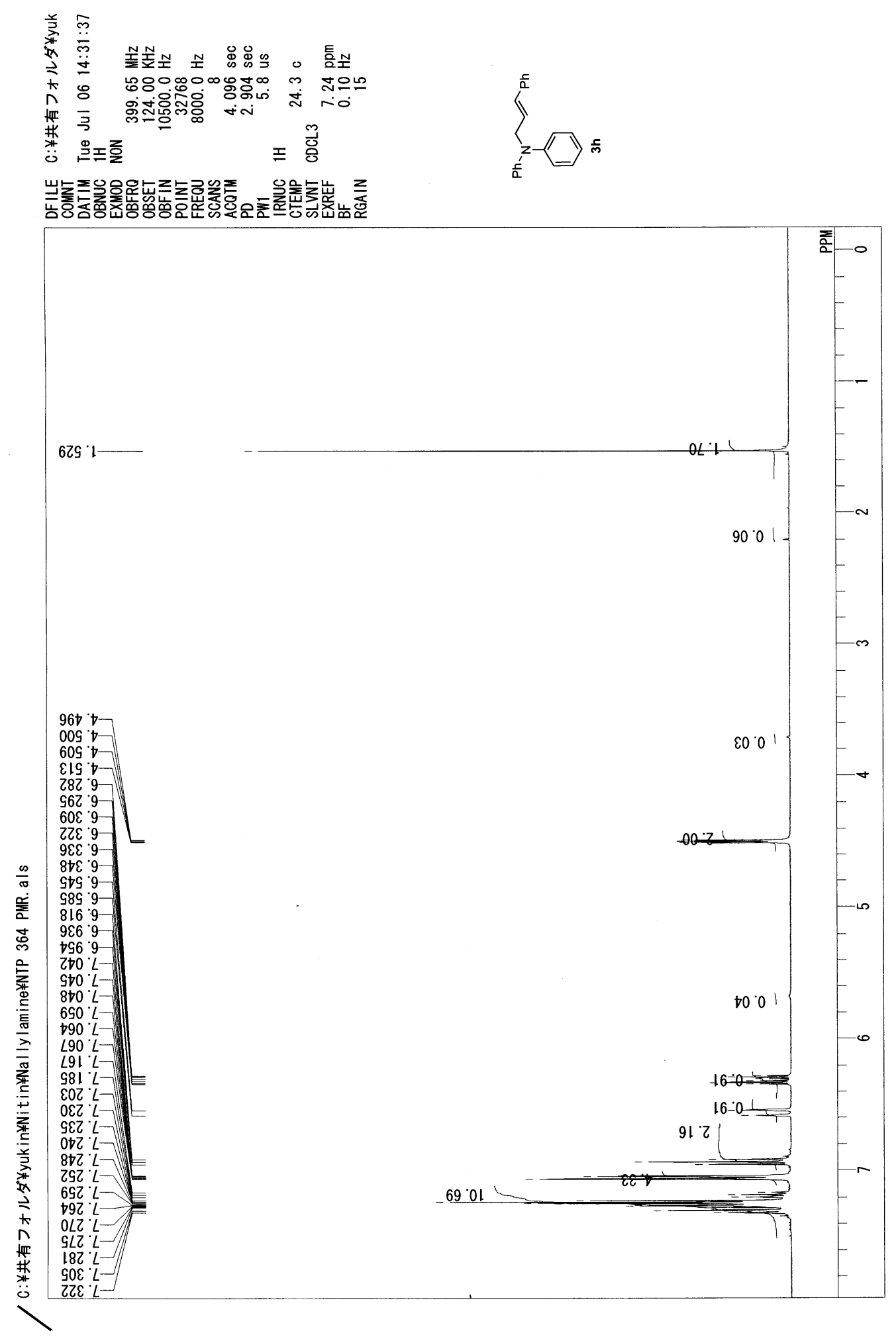




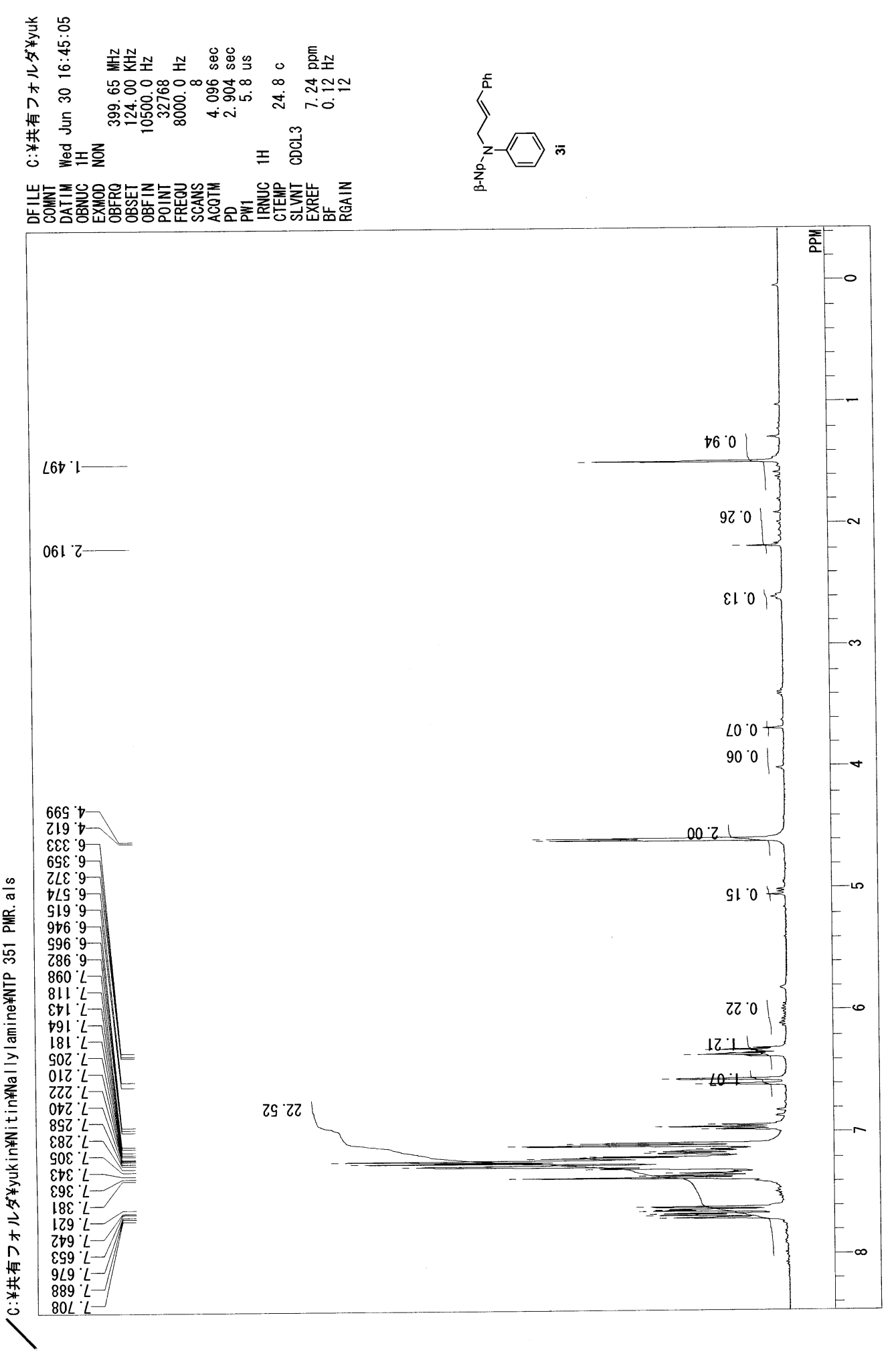




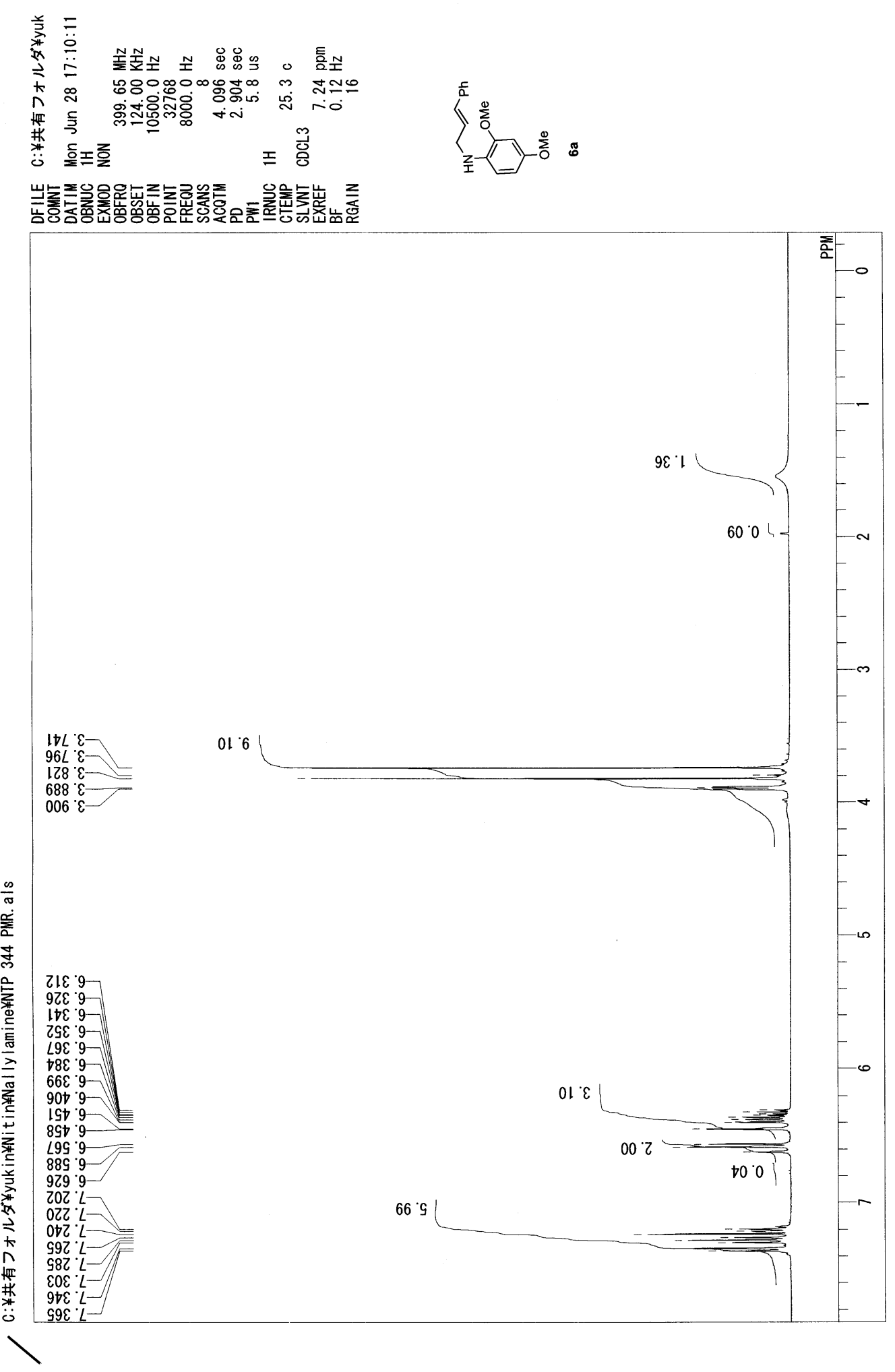




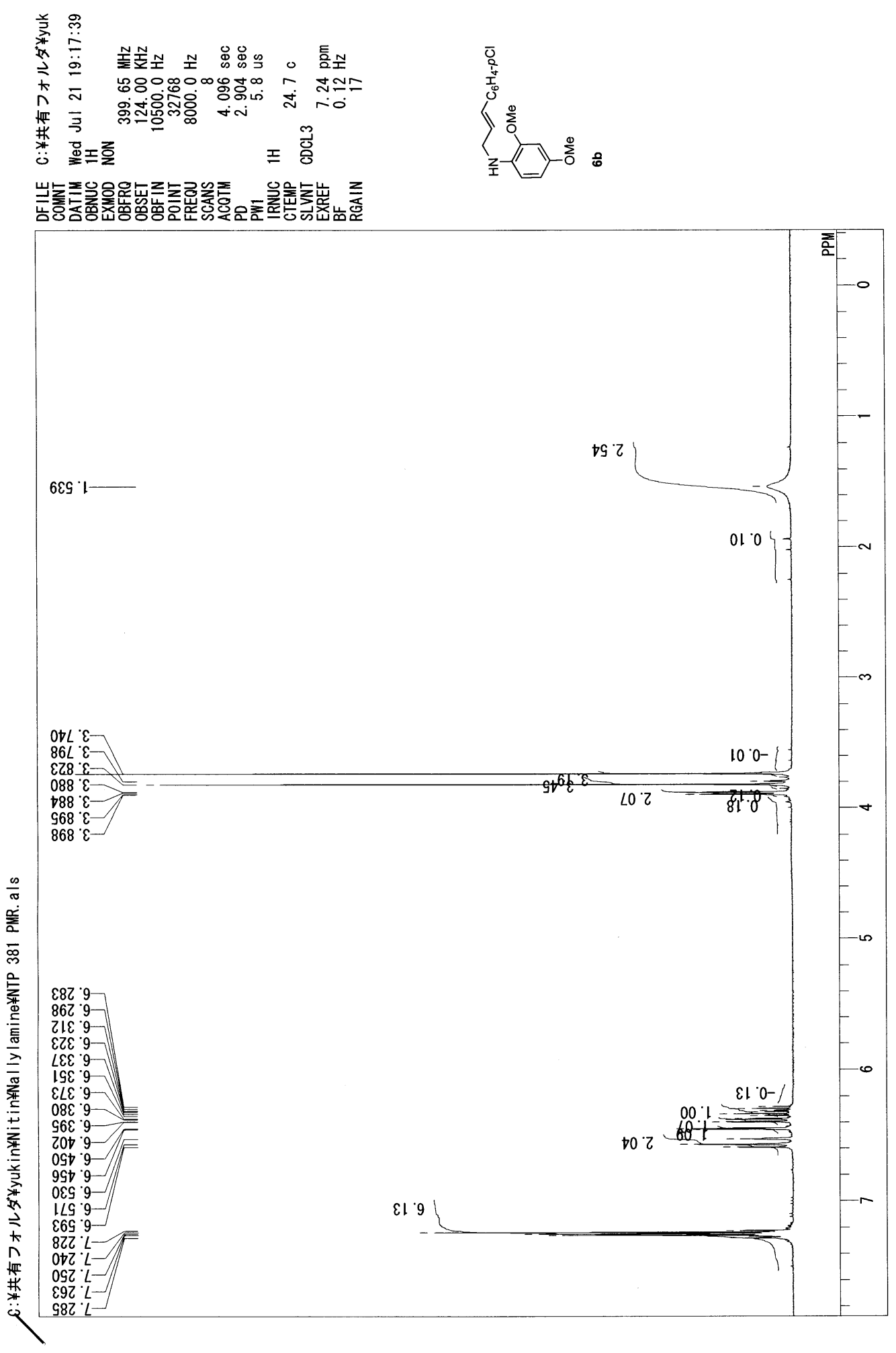




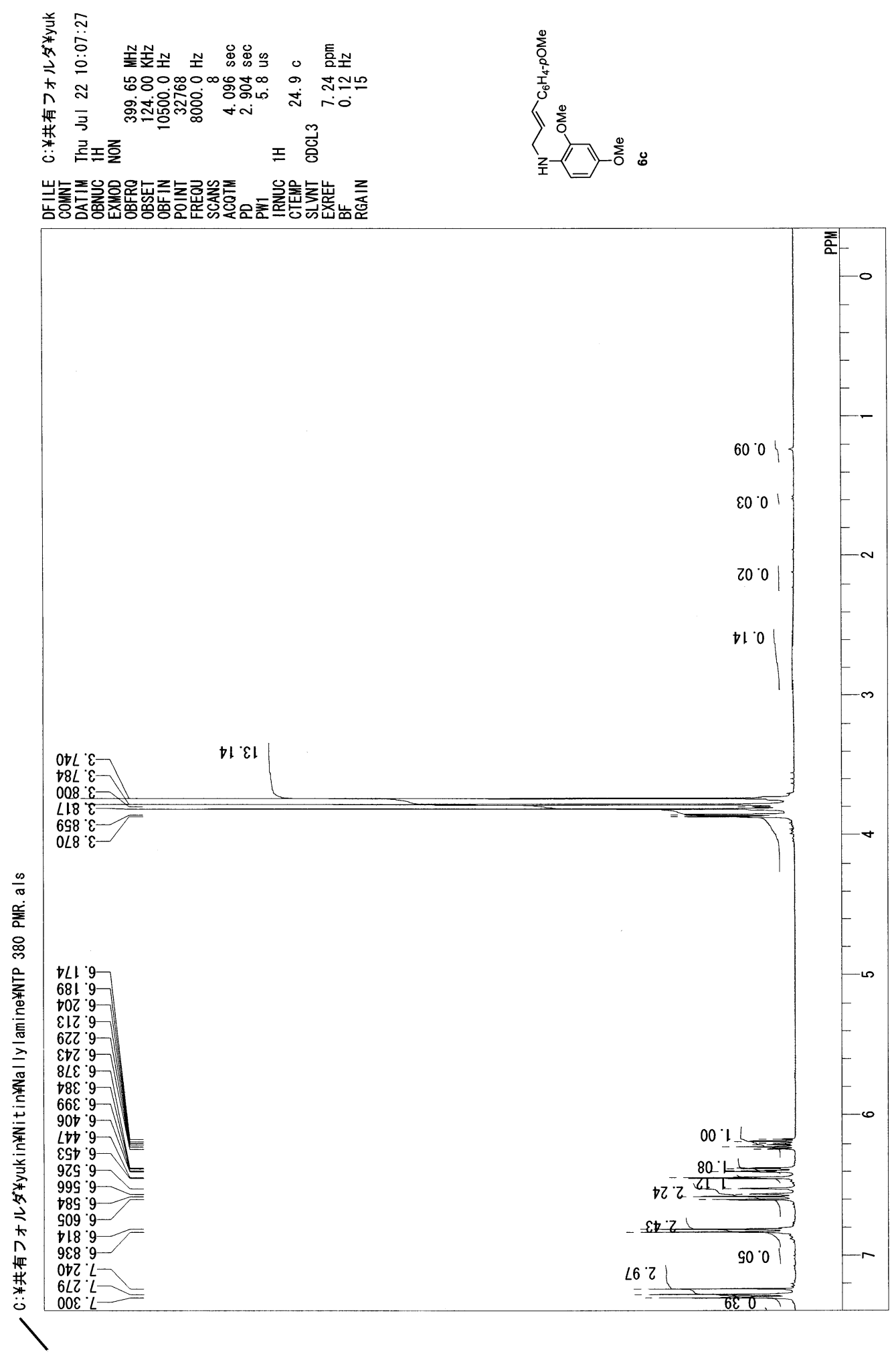




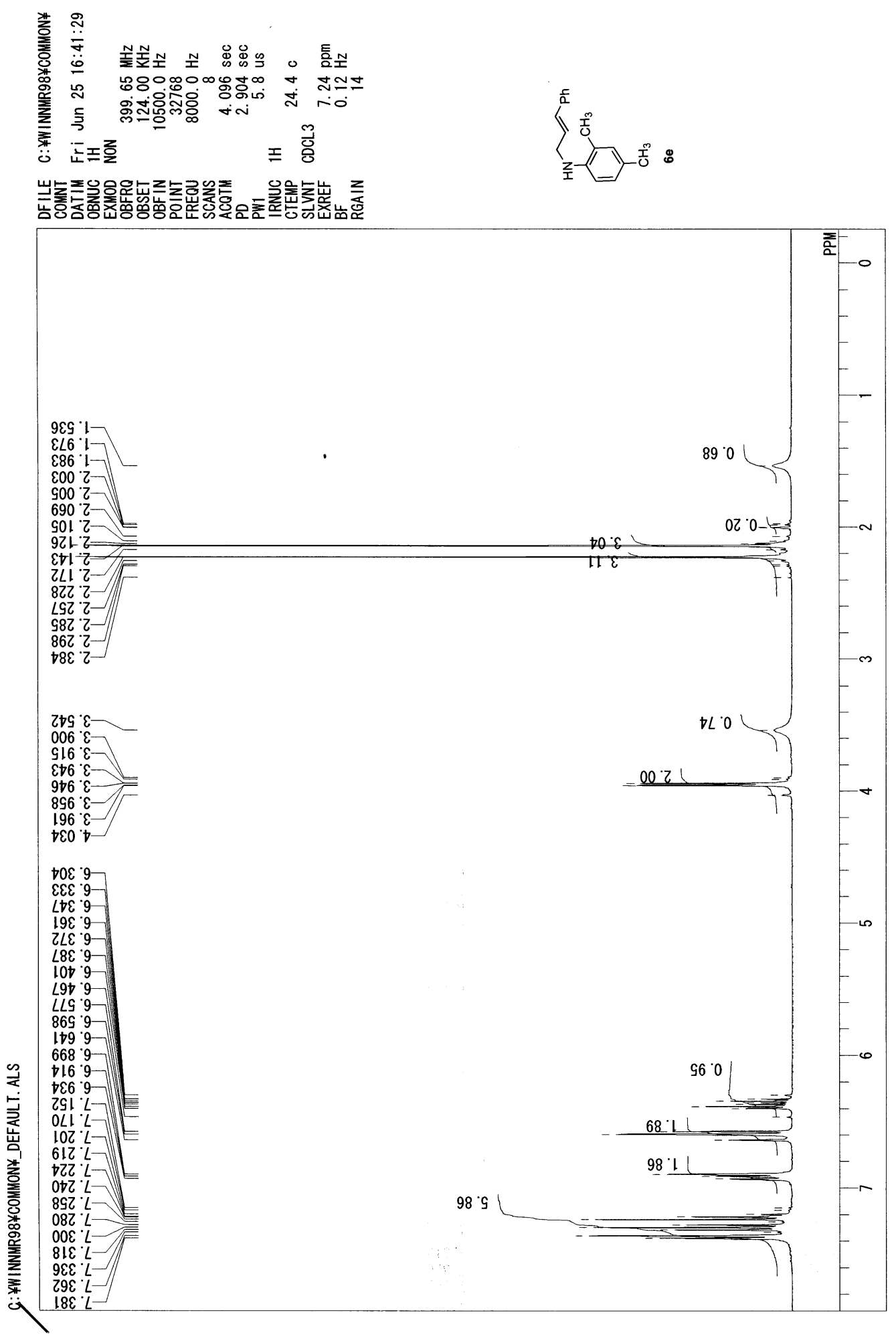




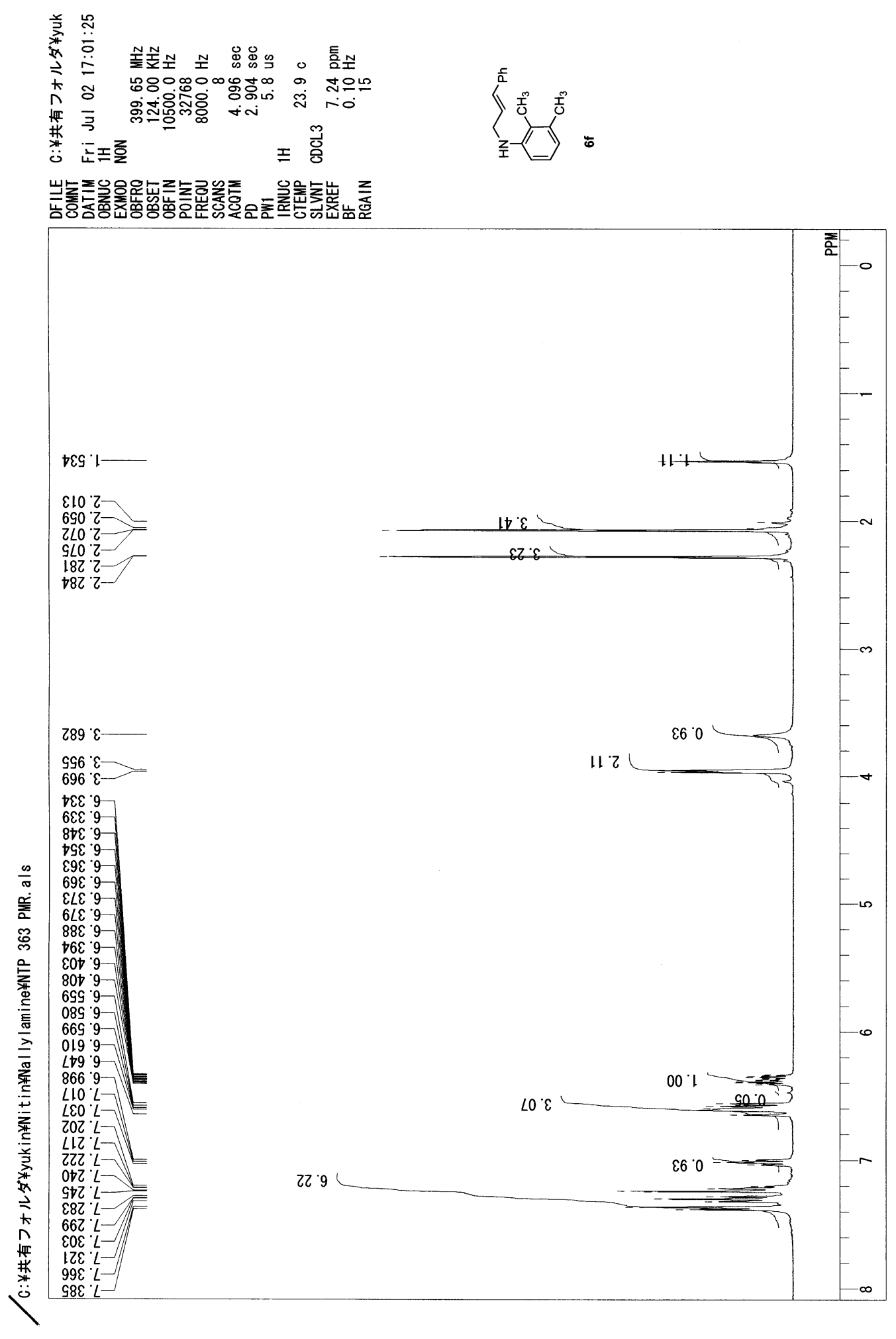




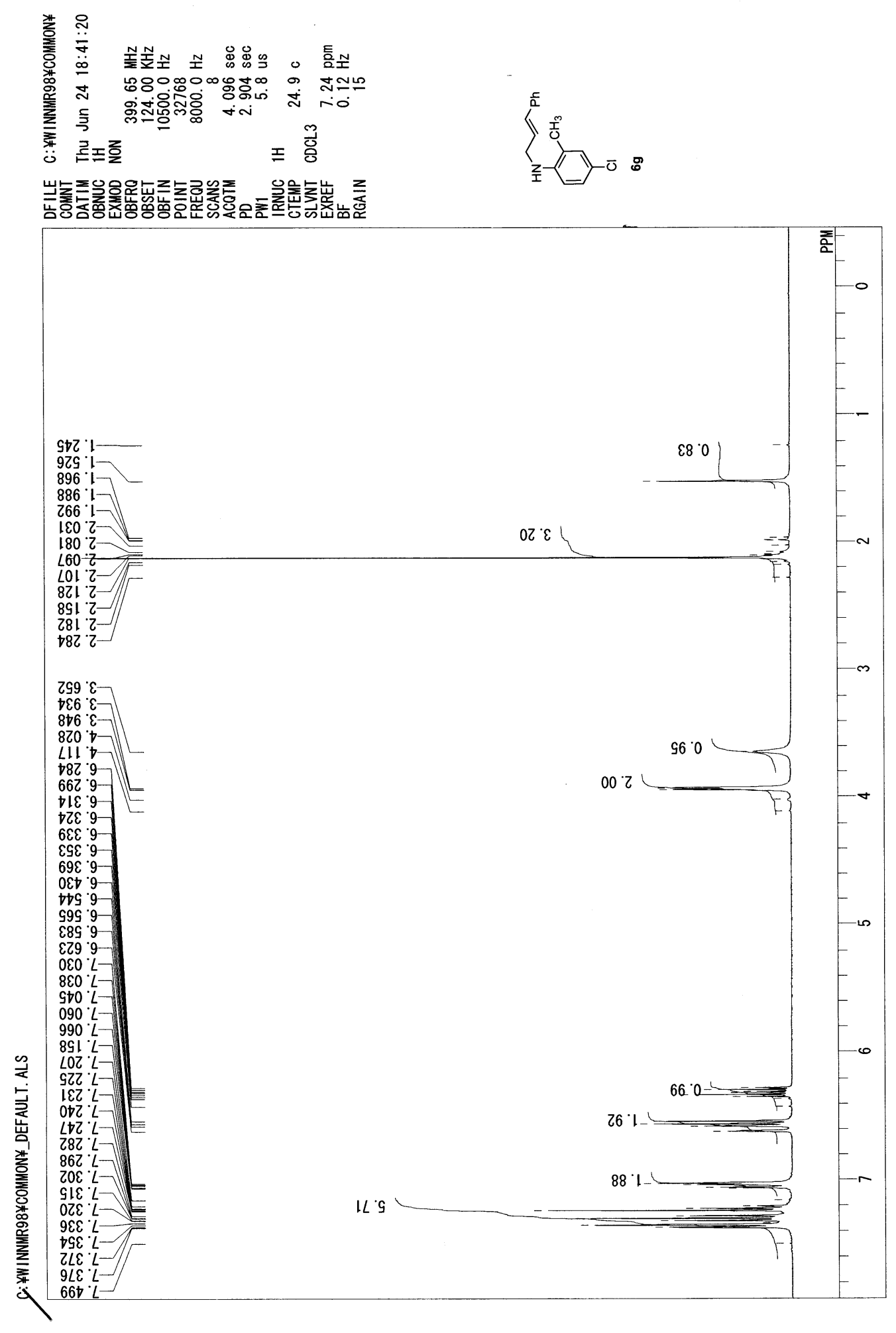




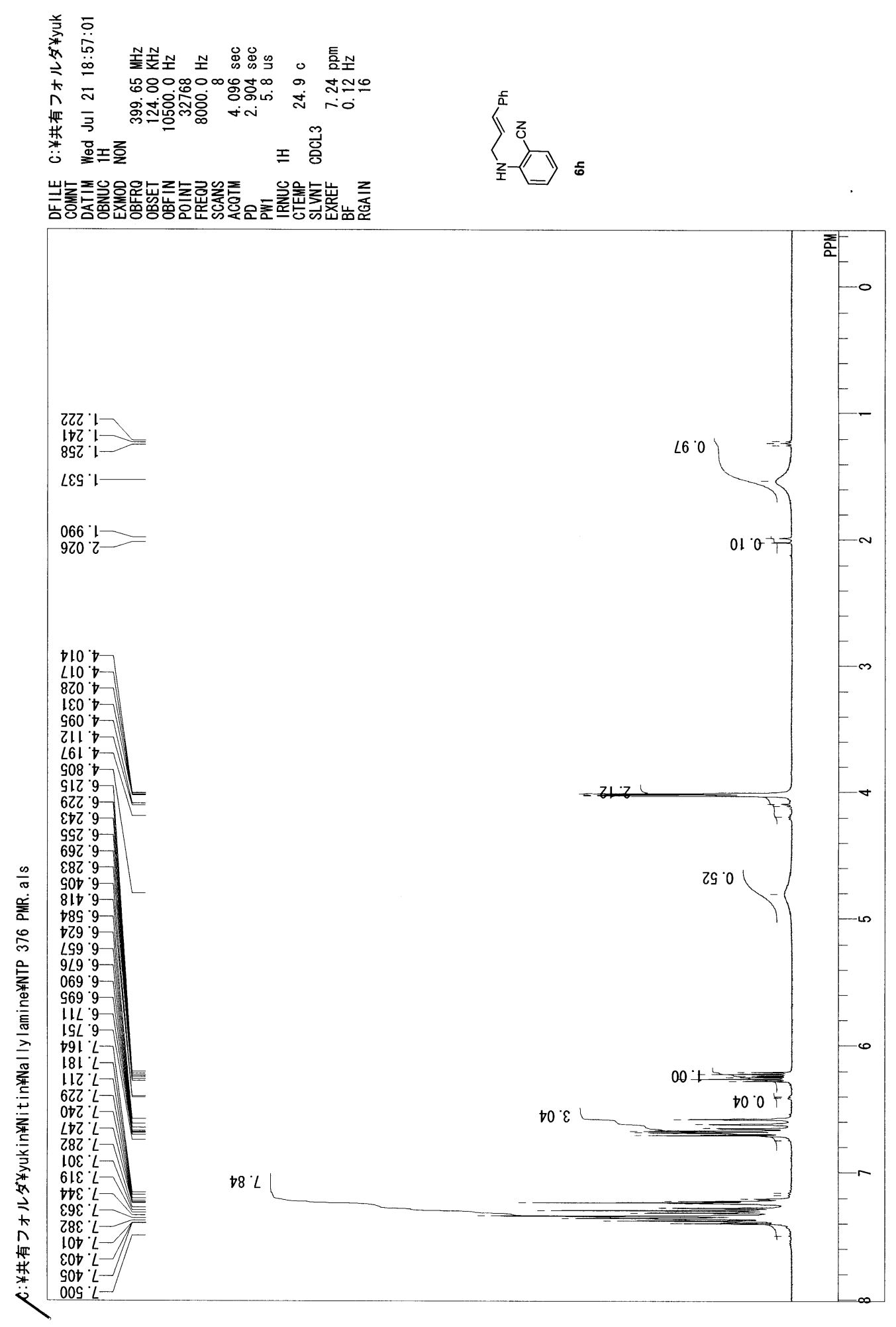



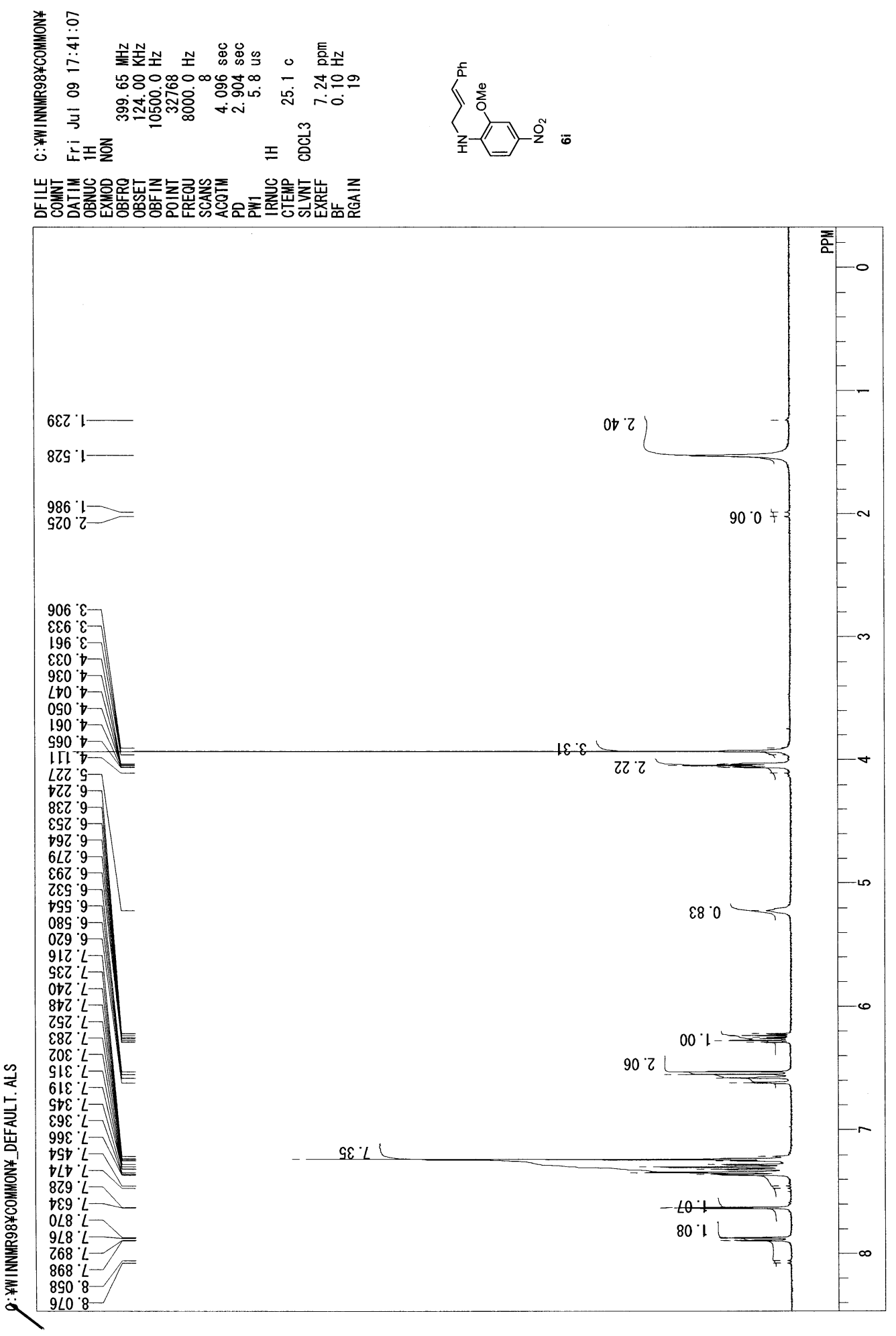


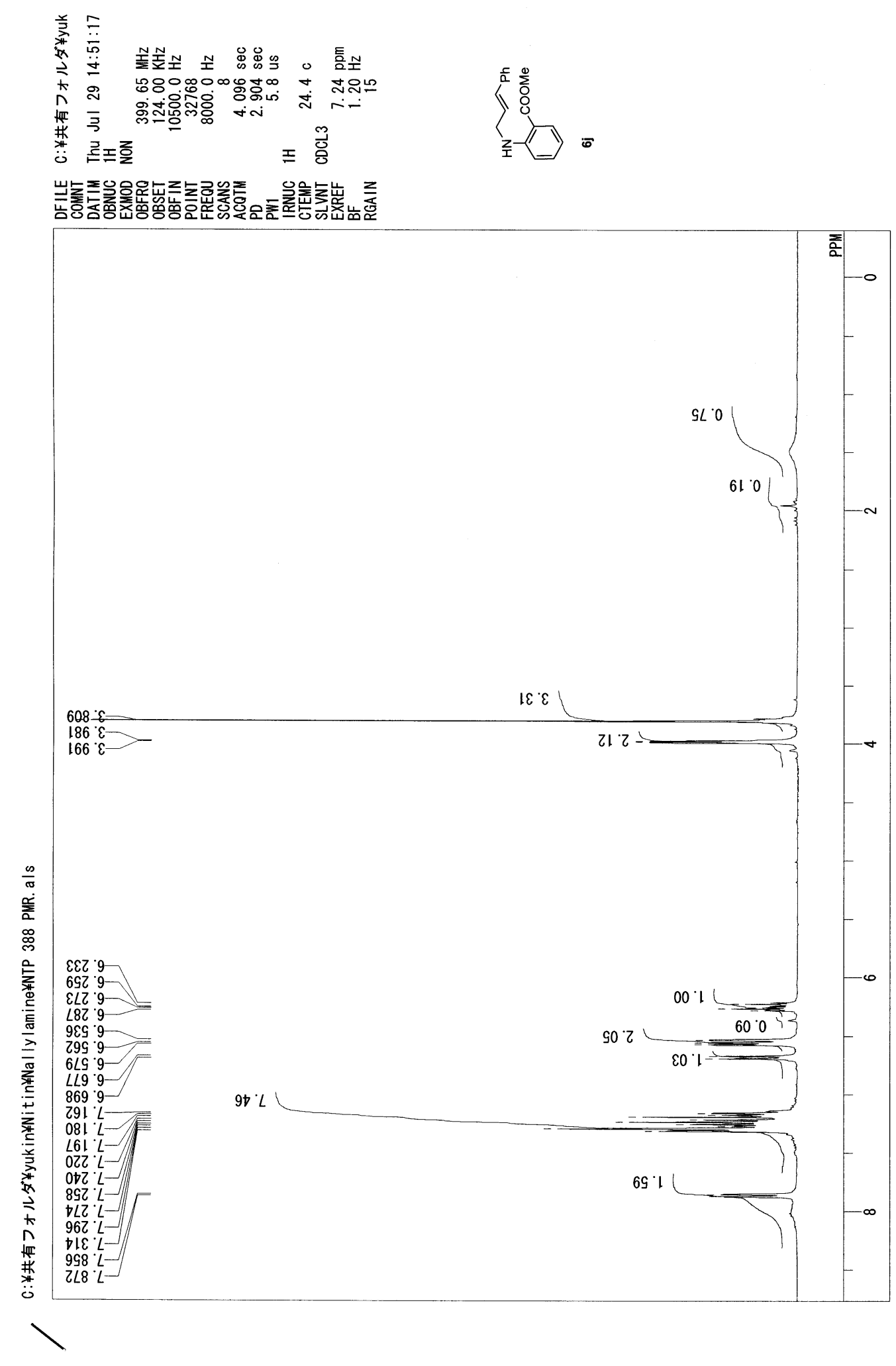




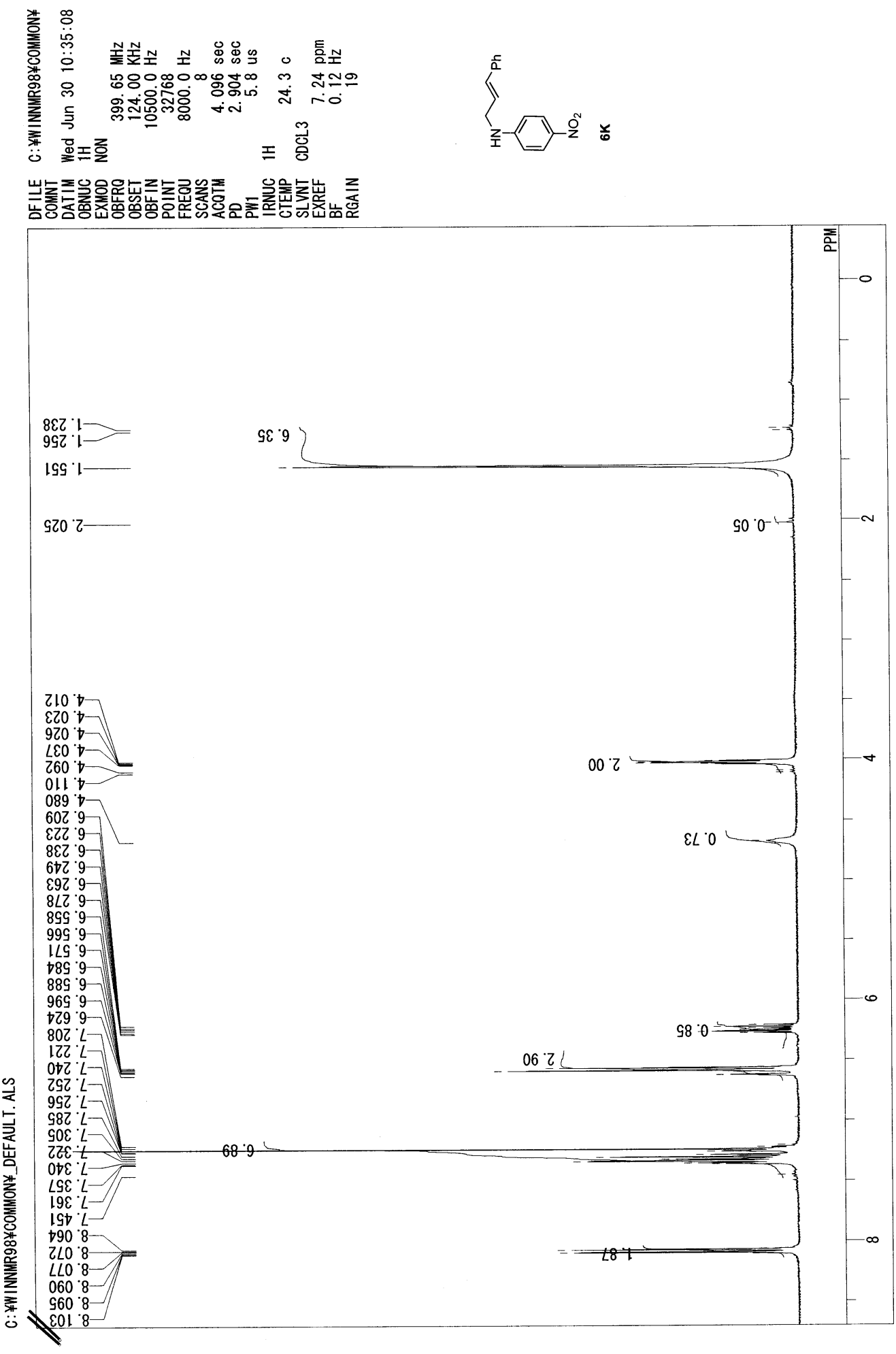




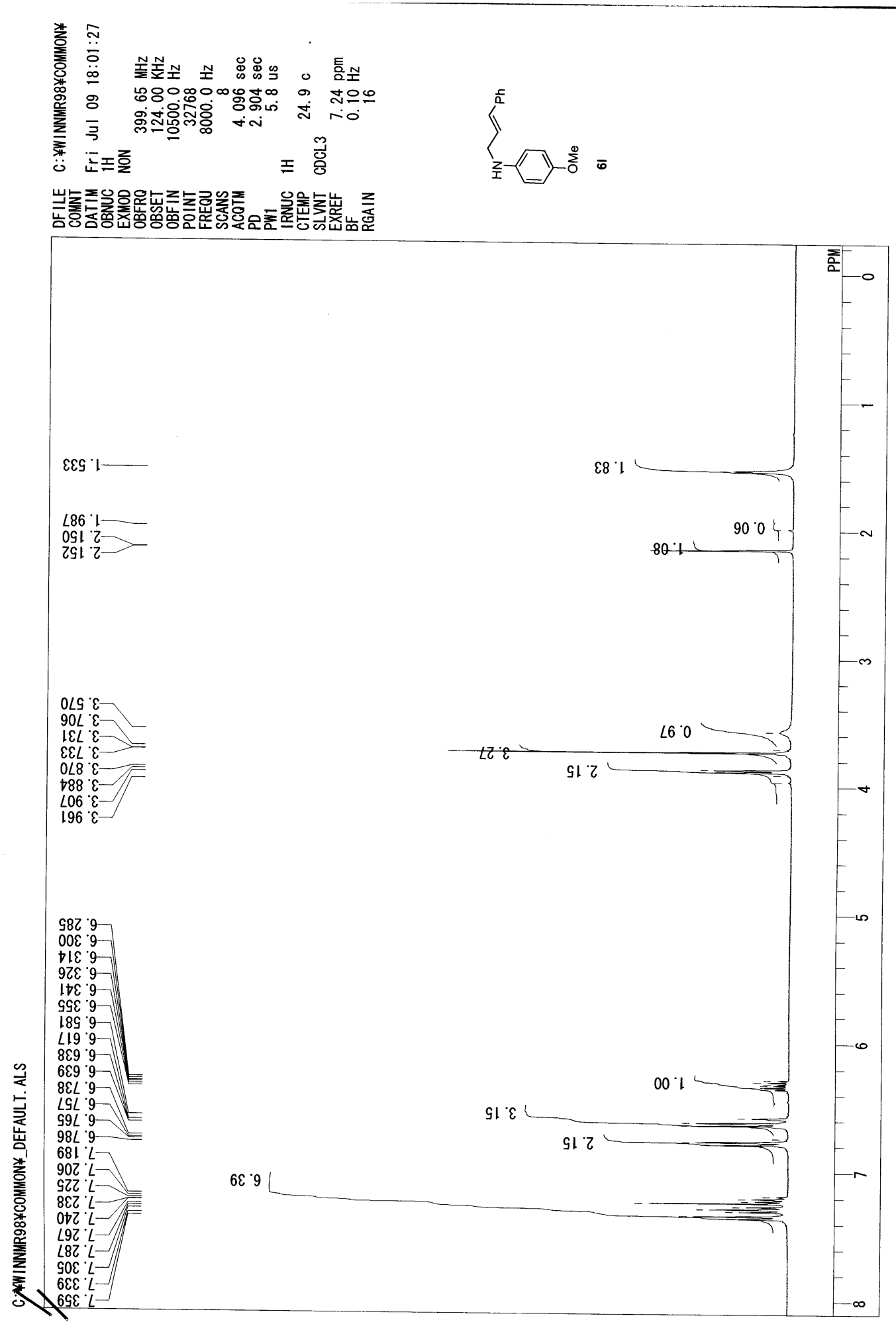




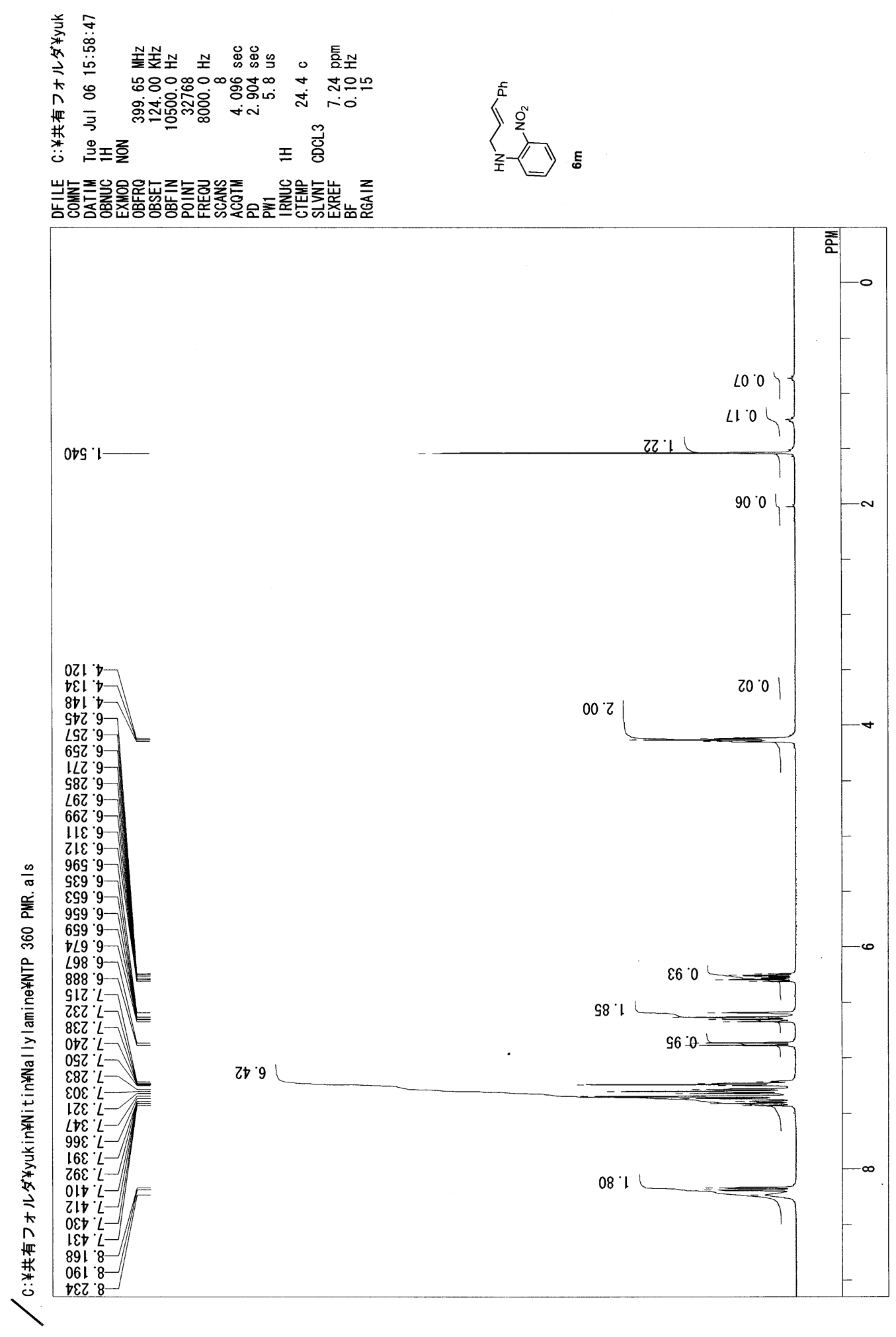




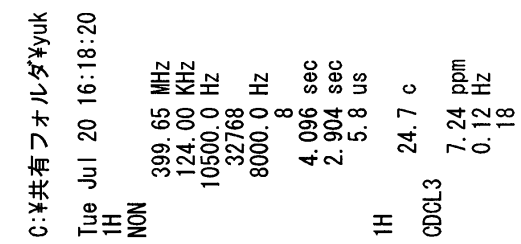

I 80

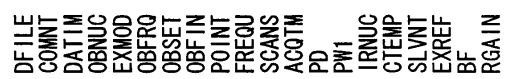

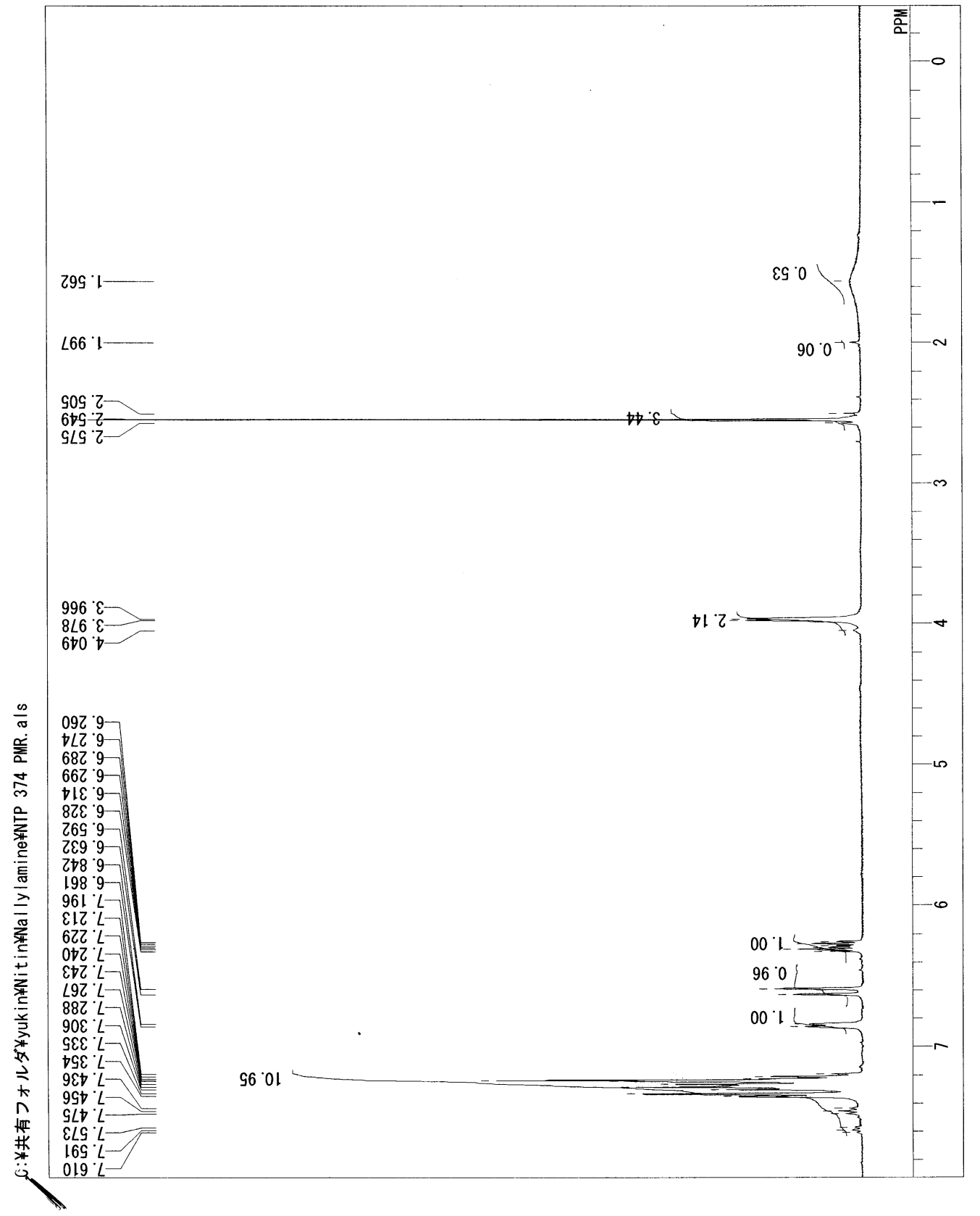




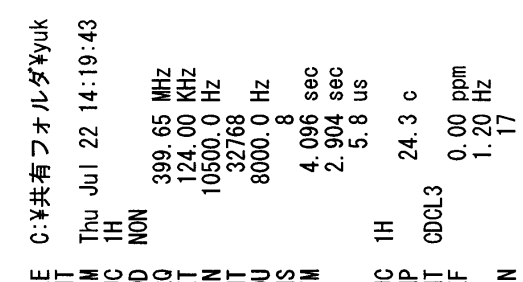

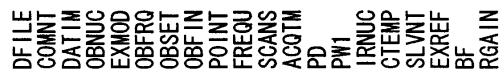
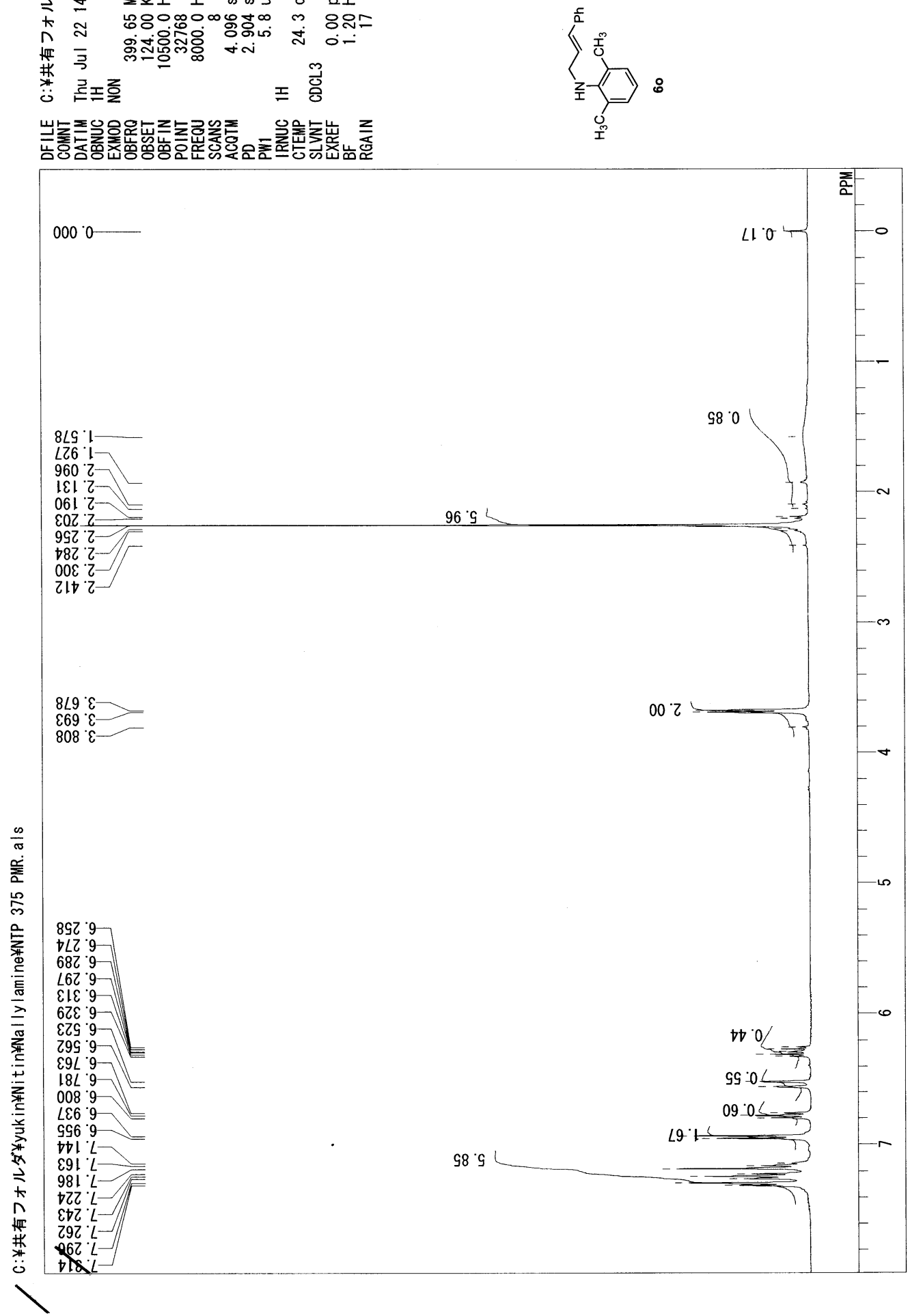


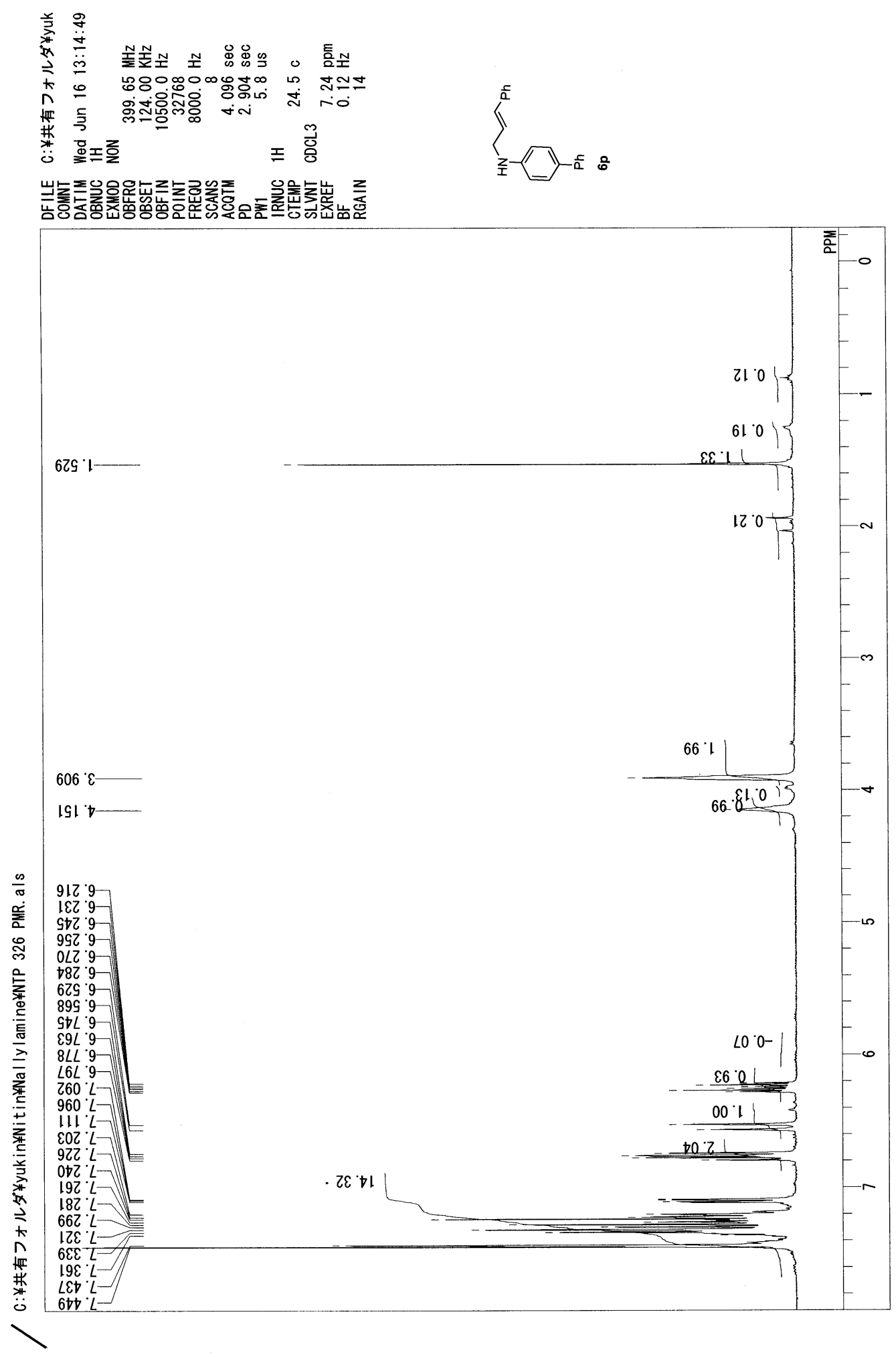




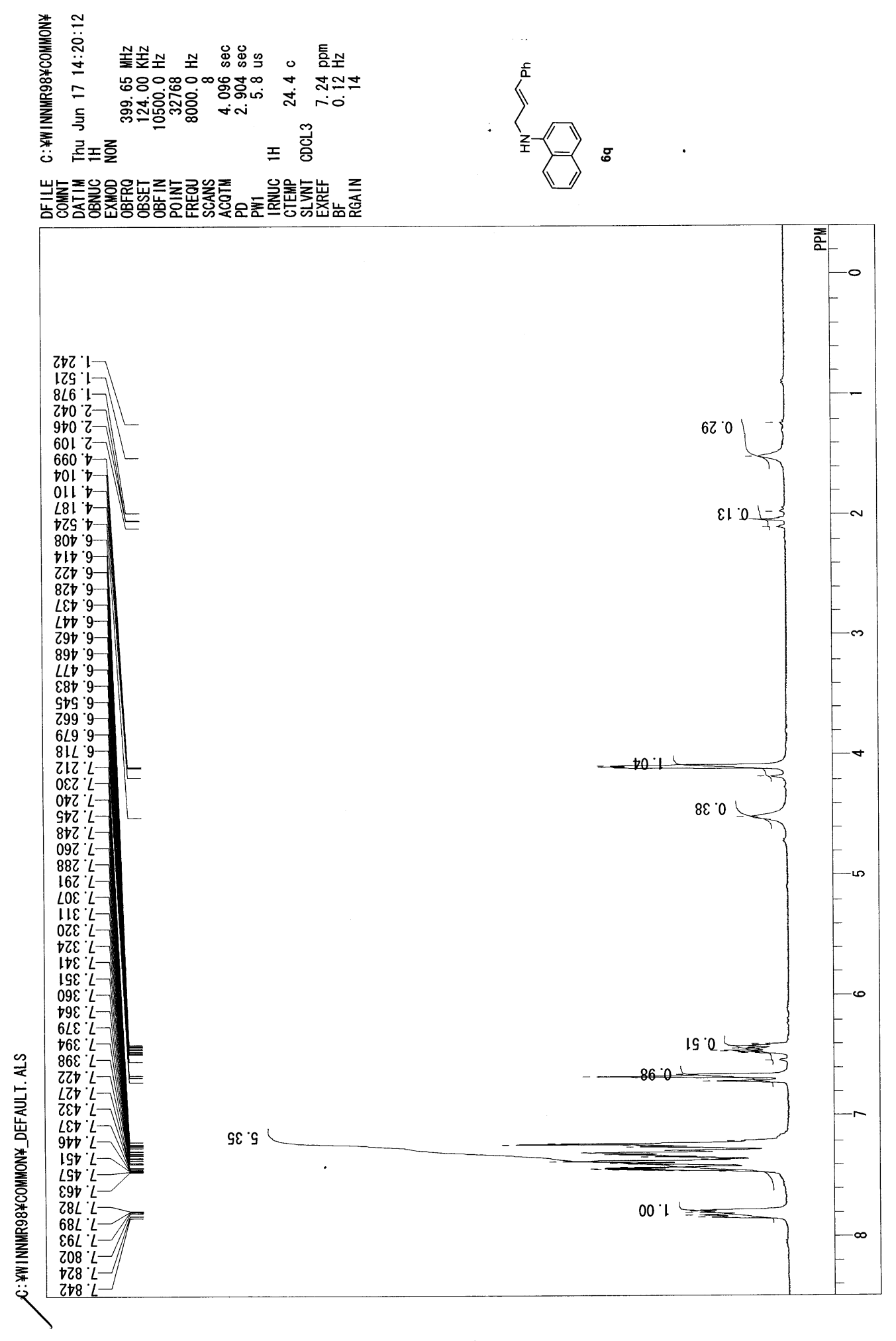




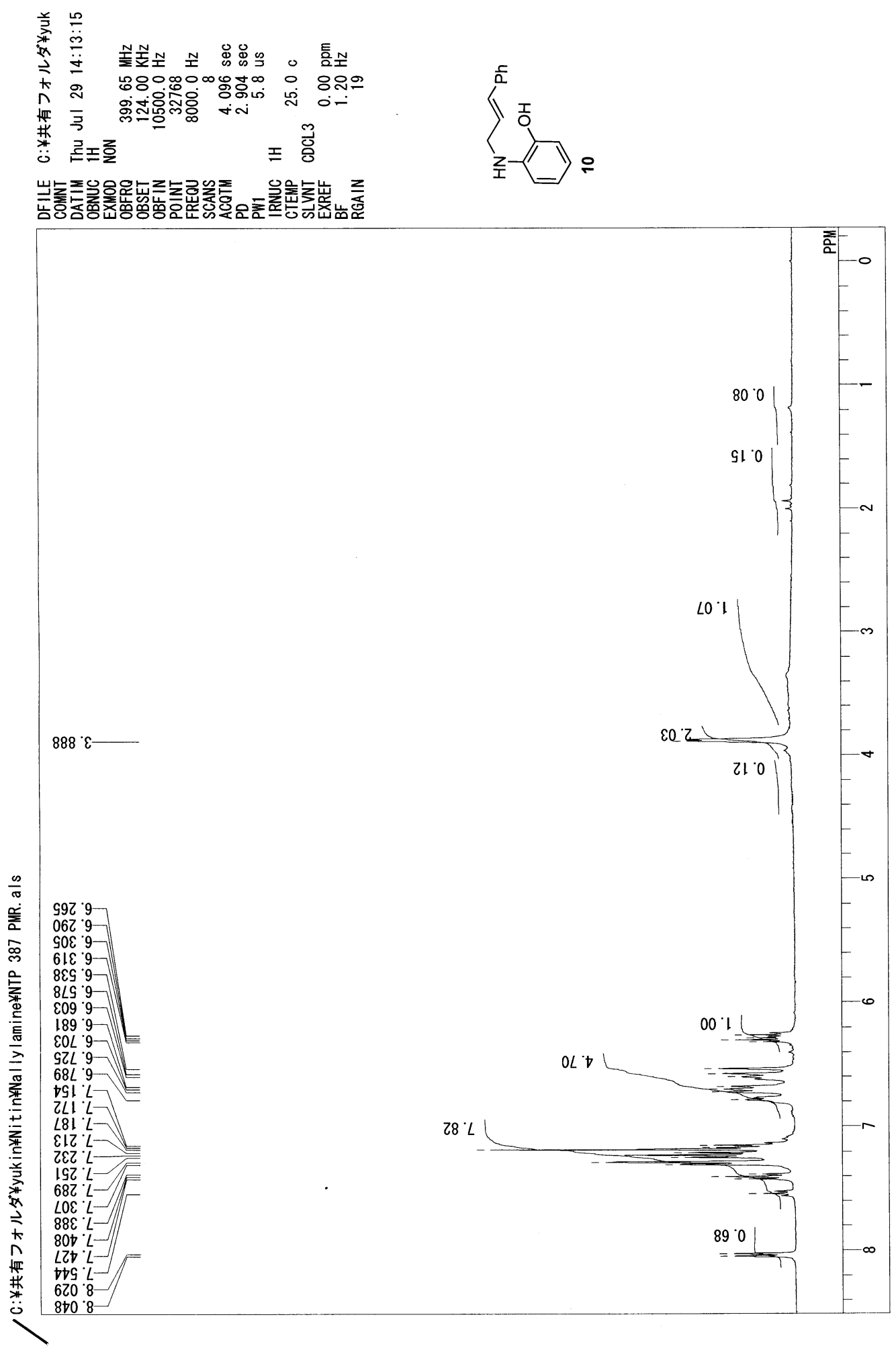


General Procedure for the $\alpha$-allylation of aldehydes: The reaction of the aldehyde 11a with 1phenyl-1-propyne 2a is representative. To a mixture of $\mathbf{1 1 a}(0.100 \mathrm{~g}, 0.745 \mathrm{mmol})$, 1-phenyl-1propyne 2a $(0.087 \mathrm{~g}, 0.745 \mathrm{mmol})$, and $\mathrm{Pd}\left(\mathrm{PPh}_{3}\right)_{4}(0.043 \mathrm{~g}, 0.037 \mathrm{mmol})$ in dry 1,4-dioxane $(2 \mathrm{~mL})$ was added benzoic acid $(0.009 \mathrm{~g}, 0.075 \mathrm{mmol})$, and the mixture was stirred at $100{ }^{\circ} \mathrm{C}$ for $15 \mathrm{~h}$. The reaction mixture was then filtered through a short silica gel column using ether as an eluent, and the filtrate was concentrated. The residue was purified by silica gel column chromatography (hexane/AcOEt, 9:1) to give 12a $(0.179 \mathrm{~g}, 96 \%)$.

Structures of $\mathbf{1 2} \mathbf{a},{ }^{1} \mathbf{1 2} \mathbf{i}^{2}$ are known in the literature. The characterization data for $\mathbf{1 2} \mathbf{a}, \mathbf{1 2} \mathbf{i}$ along with the newly synthesized compounds $\mathbf{1 2 b}, \mathbf{1 2 c}, \mathbf{1 2 d}, \mathbf{1 2 e}, \mathbf{1 2 f}, \mathbf{1 2 g}, \mathbf{1 2 h}, \mathbf{1 2 j}$ and the copies of their ${ }^{1} \mathrm{H}$ NMR spectra given below.

(E)-2-methyl-2,5-diphenylpent-4-enal (12a). Oil; IR (neat) 1724, $1598 \mathrm{~cm}^{-1}$; ${ }^{1} \mathrm{H} \mathrm{NMR}\left(\mathrm{CDCl}_{3}\right.$, $400 \mathrm{MHz}) \delta 9.56(\mathrm{~s}, 1 \mathrm{H}), 7.42-7.18(\mathrm{~m}, 10 \mathrm{H}), 6.39(\mathrm{dt}, J=15.5,1.1 \mathrm{~Hz}, 1 \mathrm{H}), 5.93(\mathrm{dt}, J=15.5,7.5$ $\mathrm{Hz}, 1 \mathrm{H}), 2.82$ (ddd, $J=13.2,7.5,1.1 \mathrm{~Hz}, 1 \mathrm{H}), 2.76(\mathrm{ddd}, J=13.2,7.5,1.1 \mathrm{~Hz}, 1 \mathrm{H}), 1.49$ (s, 3H); ${ }^{13} \mathrm{C} \mathrm{NMR}\left(\mathrm{CDCl}_{3}, 100 \mathrm{MHz}\right) \delta 201.7,139.4,137.2,133.6,128.9,128.4,127.4,127.2,127.1,126.9$, 126.1, 54.1, 40.0, 19.0; HRMS calcd for $\mathrm{C}_{18} \mathrm{H}_{18} \mathrm{O}\left(\mathrm{M}^{+}\right)$250.1358, found 250.1352.

(E)-5-(4-chlorophenyl)-2-methyl-2-phenylpent-4-enal (12b). Oil; IR (neat) 1716, $1598 \mathrm{~cm}^{-1} ;{ }^{1} \mathrm{H}$ $\operatorname{NMR}\left(\mathrm{CDCl}_{3}, 400 \mathrm{MHz}\right) \delta 9.59(\mathrm{~s}, 1 \mathrm{H}), 7.46-7.18(\mathrm{~m}, 9 \mathrm{H}), 6.37(\mathrm{~d}, J=16.0 \mathrm{~Hz}, 1 \mathrm{H}), 5.94(\mathrm{dt}, J=$ 16.0, 8.0 Hz, 1H), $2.85(\mathrm{dd}, J=14.0,8.0 \mathrm{~Hz}, 1 \mathrm{H}), 2.79(\mathrm{dd}, J=14.0,8.0 \mathrm{~Hz}, 1 \mathrm{H}), 1.52(\mathrm{~s}, 3 \mathrm{H}) ;{ }^{13} \mathrm{C}$ NMR $\left(\mathrm{CDCl}_{3}, 100 \mathrm{MHz}\right) \delta 201.7,139.2,135.6,132.7,132.3,128.9,128.5,127.4,127.2,127.1$, 125.6, 54.1, 39.9, 18.9; HRMS calcd for $\mathrm{C}_{18} \mathrm{H}_{17} \mathrm{ClO}\left(\mathrm{M}^{+}\right)$284.0968, found 284.0962.

(E)-5-(4-methoxyphenyl)-2-methyl-2-phenylpent-4-enal (12c). Oil; IR (neat) 1724, $1606 \mathrm{~cm}^{-1} ;{ }^{1} \mathrm{H}$ $\operatorname{NMR}\left(\mathrm{CDCl}_{3}, 400 \mathrm{MHz}\right) \delta 9.56(\mathrm{~s}, 1 \mathrm{H}), 7.41-7.16(\mathrm{~m}, 7 \mathrm{H}), 6.80(\mathrm{~m}, 2 \mathrm{H}), 6.33(\mathrm{dt}, J=15.6,1.1 \mathrm{~Hz}$, 1H), $5.80(\mathrm{dt}, J=15.6,7.5 \mathrm{~Hz}, 1 \mathrm{H}), 3.78$ (s, 3H), 2.78 (ddd, J = 13.4, 7.5, $1.1 \mathrm{~Hz}, 1 \mathrm{H}), 2.76$ (ddd, $J$ $=13.4,7.5,1.1 \mathrm{~Hz}, 1 \mathrm{H}), 1.48(\mathrm{~s}, 3 \mathrm{H}) ;{ }^{13} \mathrm{C} \mathrm{NMR}\left(\mathrm{CDCl}_{3}, 100 \mathrm{MHz}\right) \delta 201.2,158.8,139.5,132.9$, 129.9, 128.8, 127.3, 127.1, 127.0, 122.5, 113.8, 55.3, 54.2, 39.9, 19.1; HRMS calcd for $\mathrm{C}_{19} \mathrm{H}_{22} \mathrm{O}_{2}$ $\left(\mathrm{M}^{+}\right)$280.1463, found 280.1458. 
(E)-ethyl 5-formyl-5-phenylhex-2-enoate (12d). Oil; IR (neat) 1724, 1720, $1610 \mathrm{~cm}^{-1}$; ${ }^{1} \mathrm{H}$ NMR $\left(\mathrm{CDCl}_{3}, 400 \mathrm{MHz}\right) \delta 9.47(\mathrm{~s}, 1 \mathrm{H}), 7.41-7.22(\mathrm{~m}, 5 \mathrm{H}), 6.67(\mathrm{dt}, J=15.2,8.0 \mathrm{~Hz}, 1 \mathrm{H}), 5.81(\mathrm{~d}, J=$ $15.2 \mathrm{~Hz}, 1 \mathrm{H}), 4.13(\mathrm{q}, J=7.2 \mathrm{~Hz}, 2 \mathrm{H}), 2.80(\mathrm{dd}, J=14.0,8.0 \mathrm{~Hz}, 1 \mathrm{H}), 2.73(\mathrm{dd}, J=14.0,8.0 \mathrm{~Hz}$, $1 \mathrm{H}), 1.48(\mathrm{~s}, 3 \mathrm{H}), 1.23(\mathrm{t}, J=7.2 \mathrm{~Hz}, 3 \mathrm{H}) ;{ }^{13} \mathrm{C} \mathrm{NMR}\left(\mathrm{CDCl}_{3}, 100 \mathrm{MHz}\right) \delta 200.6,165.5,143.5$, 132.0, 129.0, 128.5, 125.8, 124.2, 61.8, 60.3, 42.2, 26.6, 18.17; HRMS calcd for $\mathrm{C}_{15} \mathrm{H}_{18} \mathrm{O}_{3}\left(\mathrm{M}^{+}\right)$ 246.1256 , found 246.1253 .

(E)-5-(4-(trifluoromethyl)phenyl)-2-methyl-2-phenylpent-4-enal (12e). Oil; IR (neat) 1724, $1614 \mathrm{~cm}^{-1} ;{ }^{1} \mathrm{H}$ NMR $\left(\mathrm{CDCl}_{3}, 400 \mathrm{MHz}\right) \delta 9.48(\mathrm{~s}, 1 \mathrm{H}), 7.42(\mathrm{~d}, J=8.4 \mathrm{~Hz}, 2 \mathrm{H}), 7.36-7.18(\mathrm{~m}, 7 \mathrm{H})$, $6.33(\mathrm{~d}, J=15.6 \mathrm{~Hz}, 1 \mathrm{H}), 5.96(\mathrm{dt}, J=15.6,7.6 \mathrm{~Hz}, 1 \mathrm{H}), 2.77(\mathrm{dd}, J=14.4,7.6 \mathrm{~Hz}, 1 \mathrm{H}), 2.71$ (dd, $J=14.4,7.6 \mathrm{~Hz}, 1 \mathrm{H}), 1.43(\mathrm{~s}, 3 \mathrm{H}) ;{ }^{13} \mathrm{C}$ NMR $\left(\mathrm{CDCl}_{3}, 100 \mathrm{MHz}\right) \delta 201.5,139.0,132.2,129.2$, $128.9,127.9,127.5,127.1,126.2,125.4,125.3,54.1,40.0,19.0 ;$ HRMS calcd for $\mathrm{C}_{19} \mathrm{H}_{17} \mathrm{~F}_{3} \mathrm{O}\left(\mathrm{M}^{+}\right)$ 318.1231 , found 318.1226 .

(E)-2-methyl-5-phenyl-2-p-tolylpent-4-enal (12f). Oil; IR (neat) 1724, $1610 \mathrm{~cm}^{-1} ;{ }^{1} \mathrm{H}$ NMR $\left(\mathrm{CDCl}_{3}, 400 \mathrm{MHz}\right) \delta 9.46(\mathrm{~s}, 1 \mathrm{H}), 7.18-7.08(\mathrm{~m}, 9 \mathrm{H}), 6.32(\mathrm{~d}, J=16.0 \mathrm{~Hz}, 1 \mathrm{H}), 5.87(\mathrm{dt}, J=16.0$, $7.6 \mathrm{~Hz}, 1 \mathrm{H}), 2.73$ (dd, $J=14.0,7.6 \mathrm{~Hz}, 1 \mathrm{H}), 2.68$ (dd, $J=14.0,7.6 \mathrm{~Hz}, 1 \mathrm{H}), 2.28$ (s, 3H), 1.39 (s, $3 \mathrm{H}) ;{ }^{13} \mathrm{C}$ NMR $\left(\mathrm{CDCl}_{3}, 100 \mathrm{MHz}\right) \delta 201.8,137.2,137.1,136.3,133.4,129.6,128.4,127.1,126.9$, 126.0, 125.0, 53.8, 39.9, 21.0, 19.0; HRMS calcd for $\mathrm{C}_{19} \mathrm{H}_{20} \mathrm{O}\left(\mathrm{M}^{+}\right)$264.1514, found 264.1509.

(E)-2-(4-methoxyphenyl)-2-methyl-5-phenylpent-4-enal (12g). Oil; IR (neat) 1716, $1608 \mathrm{~cm}^{-1}$; ${ }^{1} \mathrm{H}$ NMR $\left(\mathrm{CDCl}_{3}, 400 \mathrm{MHz}\right) \delta 9.50(\mathrm{~s}, 1 \mathrm{H}), 7.25-7.18(\mathrm{~m}, 7 \mathrm{H}), 6.92(\mathrm{~d}, J=8.4 \mathrm{~Hz}, 2 \mathrm{H}), 6.39(\mathrm{~d}, J=$ $15.6 \mathrm{~Hz}, 1 \mathrm{H}), 5.93(\mathrm{dt}, J=15.6,7.6 \mathrm{~Hz}, 1 \mathrm{H}), 3.82(\mathrm{~s}, 3 \mathrm{H}), 2.80(\mathrm{dd}, J=14.0,7.6 \mathrm{~Hz}, 1 \mathrm{H}), 2.73(\mathrm{dd}$, $J=14.0,7.6 \mathrm{~Hz}, 1 \mathrm{H}), 1.46(\mathrm{~s}, 3 \mathrm{H}) ;{ }^{13} \mathrm{C} \mathrm{NMR}\left(\mathrm{CDCl}_{3}, 100 \mathrm{MHz}\right) \delta 201.7,158.7,137.2,133.4$, 131.1, 128.4, 128.3, 127.1, 126.0, 125.0, 114.3, 55.3, 53.4, 40.0, 19.0; HRMS calcd for $\mathrm{C}_{19} \mathrm{H}_{20} \mathrm{O}_{2}$ $\left(\mathrm{M}^{+}\right)$280.1463, found 280.1458 .

(E)-2-(4-chlorophenyl)-2-methyl-5-phenylpent-4-enal (12h). Oil; IR (neat) 1717, $1609 \mathrm{~cm}^{-1} ;{ }^{1} \mathrm{H}$ NMR $\left(\mathrm{CDCl}_{3}, 400 \mathrm{MHz}\right) \delta 9.50(\mathrm{~s}, 1 \mathrm{H}), 7.36(\mathrm{~d}, J=8.4 \mathrm{~Hz}, 2 \mathrm{H}), 7.28(\mathrm{~m}, 7 \mathrm{H}), 6.40(\mathrm{~d}, J=15.6$ $\mathrm{Hz}, 1 \mathrm{H}), 5.89$ (dt, $J=15.6,7.2 \mathrm{~Hz}, 1 \mathrm{H}), 2.80(\mathrm{dd}, J=14.4,7.2 \mathrm{~Hz}, 1 \mathrm{H}), 2.74(\mathrm{dd}, J=14.4,7.2 \mathrm{~Hz}$, 
1H), $1.45(\mathrm{~s}, 3 \mathrm{H}) ;{ }^{13} \mathrm{C} \mathrm{NMR}\left(\mathrm{CDCl}_{3}, 100 \mathrm{MHz}\right) \delta 201.2,137.9,136.9,133.9,133.4,128.9,128.5$, 128.4, 127.3, 126.0, 124.2, 53.8, 40.0, 19.1; HRMS calcd for $\mathrm{C}_{18} \mathrm{H}_{17} \mathrm{ClO}\left(\mathrm{M}^{+}\right)$284.0968, found 284.0962 .

(E)-2,2,5-triphenylpent-4-enal (12i). Oil; IR (neat) 1724, $1598 \mathrm{~cm}^{-1} ;{ }^{1} \mathrm{H} \mathrm{NMR}\left(\mathrm{CDCl}_{3}, 400 \mathrm{MHz}\right)$ $\delta 9.86(\mathrm{~s}, 1 \mathrm{H}), 7.38-7.13(\mathrm{~m}, 15 \mathrm{H}), 6.26(\mathrm{~d}, J=16.0 \mathrm{~Hz}, 1 \mathrm{H}), 5.93(\mathrm{dt}, J=16.0,7.2 \mathrm{~Hz}, 1 \mathrm{H}), 3.22$

(broad doublet, $J=6.8 \mathrm{~Hz}, 2 \mathrm{H}) ;{ }^{13} \mathrm{C} \mathrm{NMR}\left(\mathrm{CDCl}_{3}, 100 \mathrm{MHz}\right) \delta 198.2,139.6,137.3,133.3,129.9$, 129.2, 129.1, 128.6, 128.3, 128.2, 128.1, 127.4, 126.9, 125.9, 125.3, 63.9, 37.9;HRMS calcd for $\mathrm{C}_{23} \mathrm{H}_{20} \mathrm{O}\left(\mathrm{M}^{+}\right) 312.1514$, found 312.1509.

(E)-5-(4-chlorophenyl)-2,2-diphenylpent-4-enal (12j). Oil; IR (neat) 1724, $1597 \mathrm{~cm}^{-1}$; ${ }^{1} \mathrm{H}$ NMR $\left(\mathrm{CDCl}_{3}, 400 \mathrm{MHz}\right) \delta 9.85(\mathrm{~s}, 1 \mathrm{H}), 7.38-7.16(\mathrm{~m}, 12 \mathrm{H}), 7.05(\mathrm{~d}, \mathrm{~J}=8.4 \mathrm{~Hz}, 2 \mathrm{H}), 6.18(\mathrm{~d}, \mathrm{~J}=15.6$ $\mathrm{Hz}, 1 \mathrm{H}), 5.90$ (dt, J = 15.6, 7.2 Hz, 1H), 3.21 (broad doublet, $\mathrm{J}=7.6 \mathrm{~Hz}, 2 \mathrm{H}) ;{ }^{13} \mathrm{C} \mathrm{NMR}\left(\mathrm{CDCl}_{3}\right.$, $100 \mathrm{MHz}) \delta 198.0,139.5,135.8,132.6,132.2$, 129.5, 129.4, 129.0, 128.7, 128.4, 128.2, 127.4 , 127.1, 126.1, 64.0, 37.9; HRMS calcd for $\mathrm{C}_{23} \mathrm{H}_{19} \mathrm{ClO}\left(\mathrm{M}^{+}\right)$346.1124, found 346.1121.

\section{References:}

1. Kimura, M.; Horino, Y.; Mukai, R.; Tanaka, S.; Tamaru Y. J. Am. Chem. Soc. 2001, 123, 10401-10402.

2. Ciganek, E.; Read, J. M.; Calabrese, J. C. J. Org. Chem. 1995, 60, 5795-5802. 

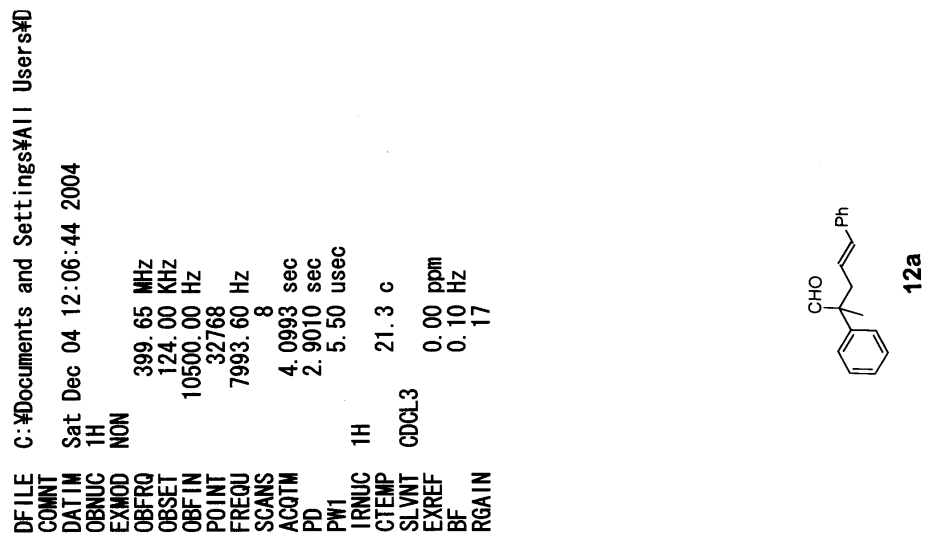

$000 \%$
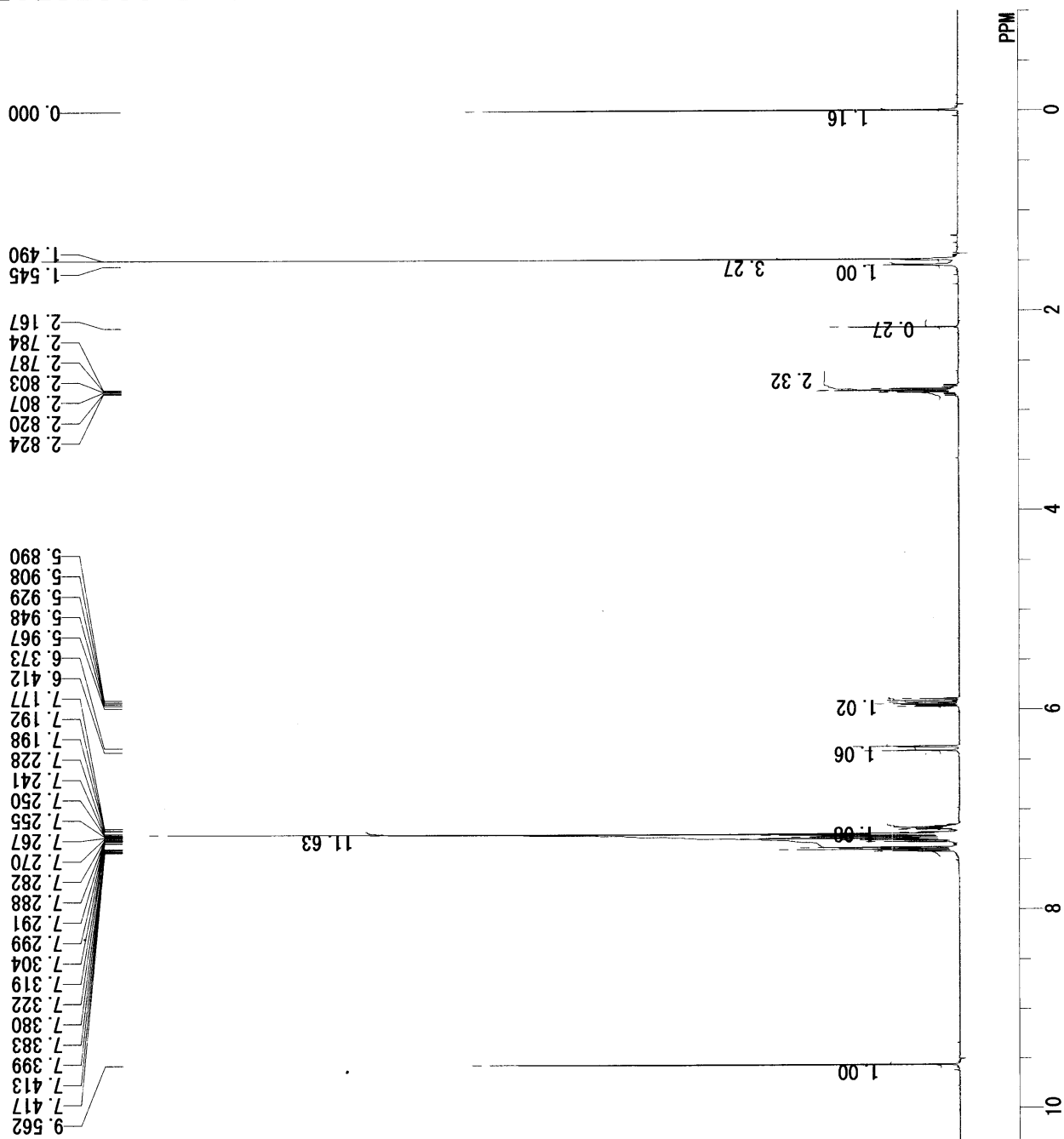

乙ะ $乙$

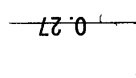

E9 11

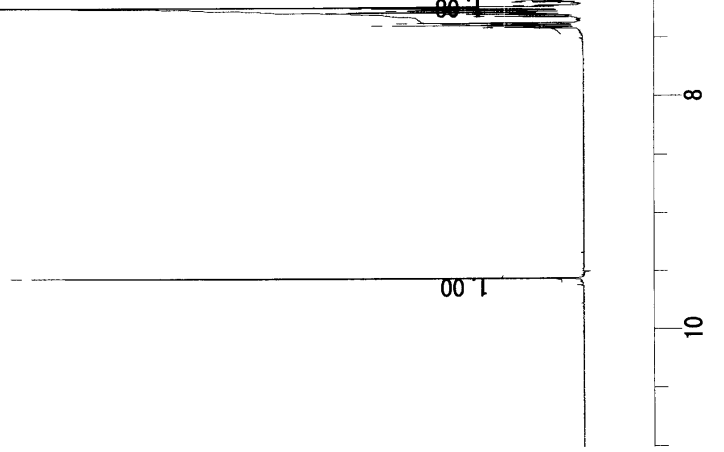



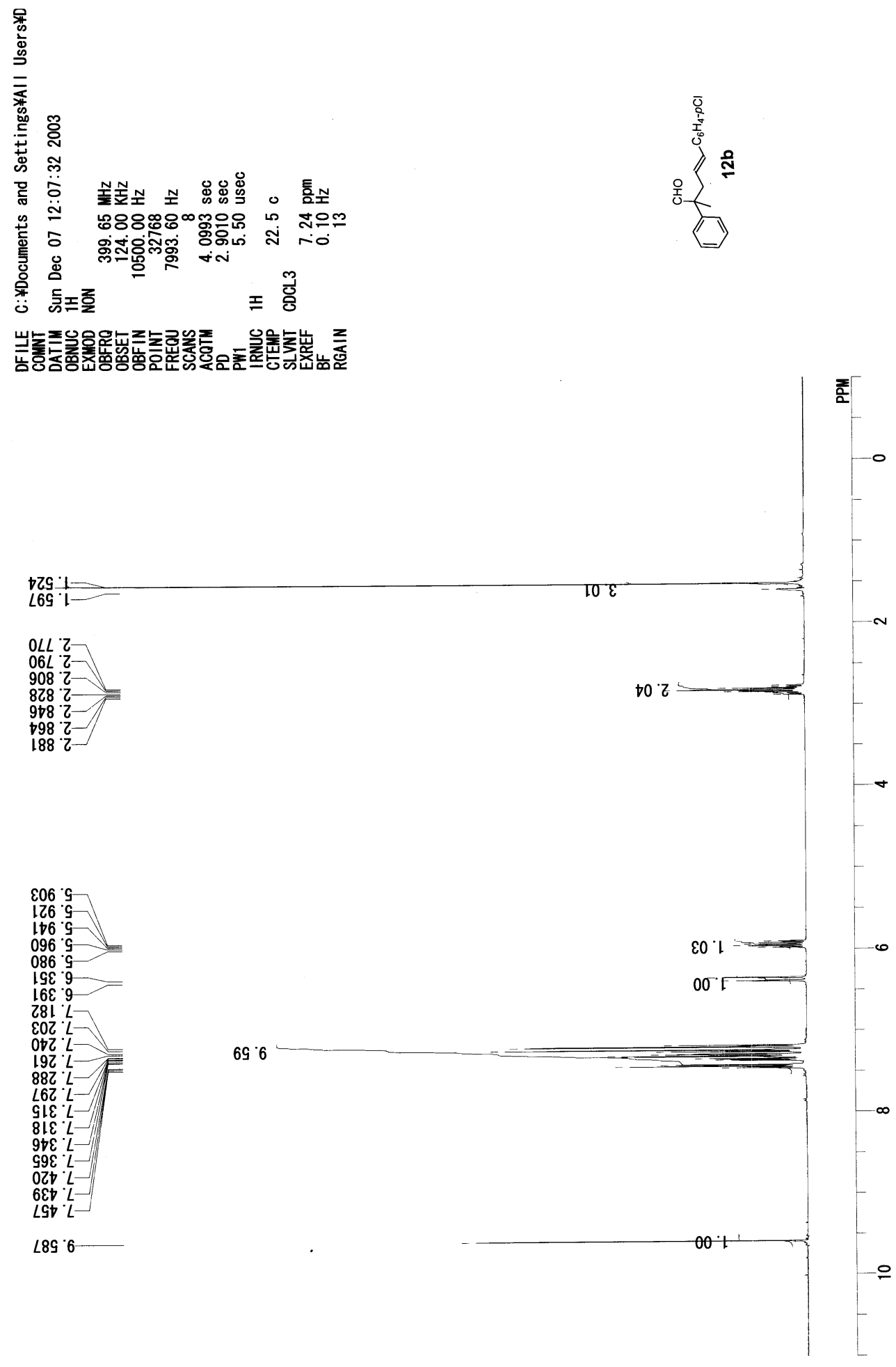

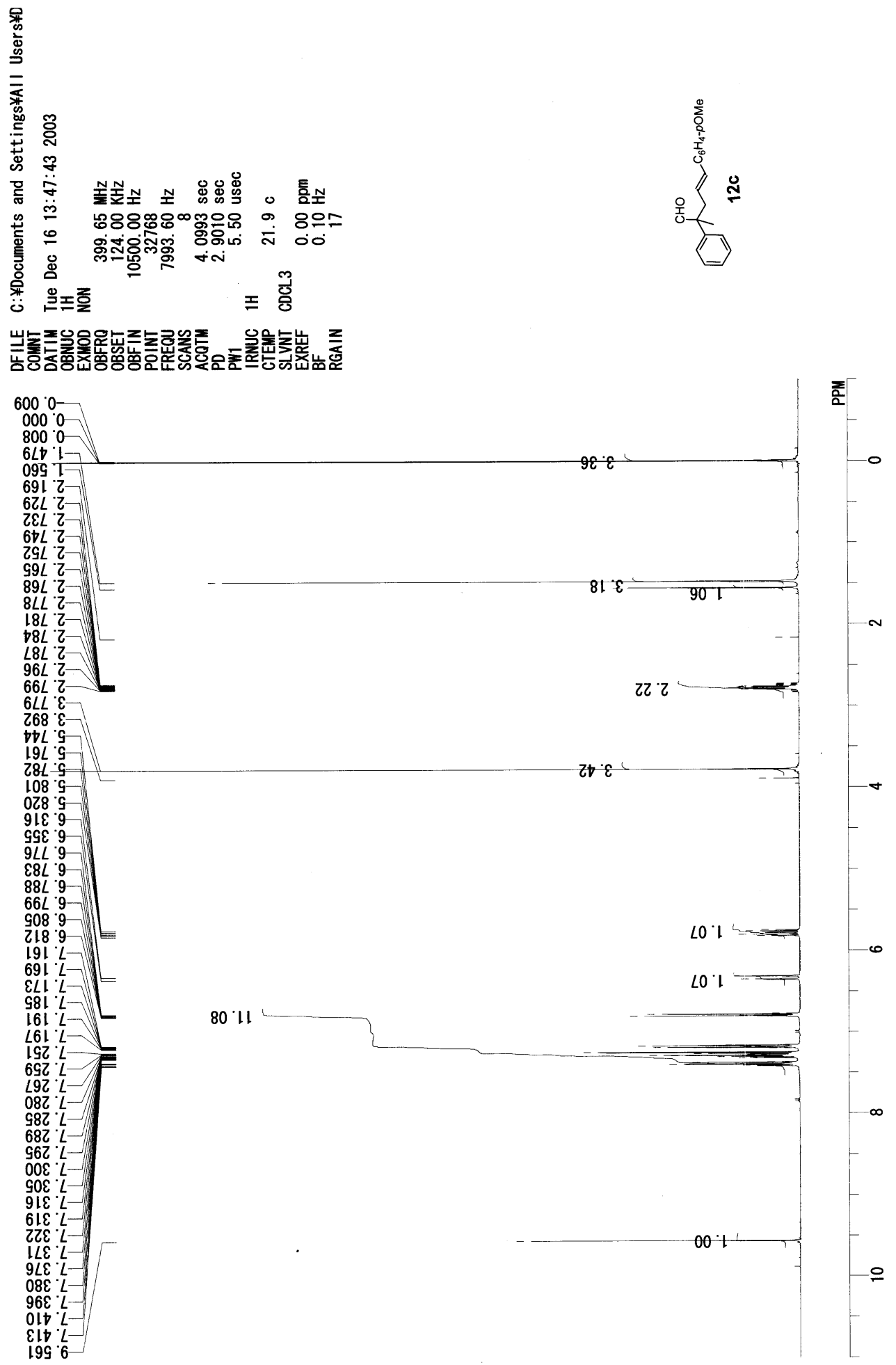

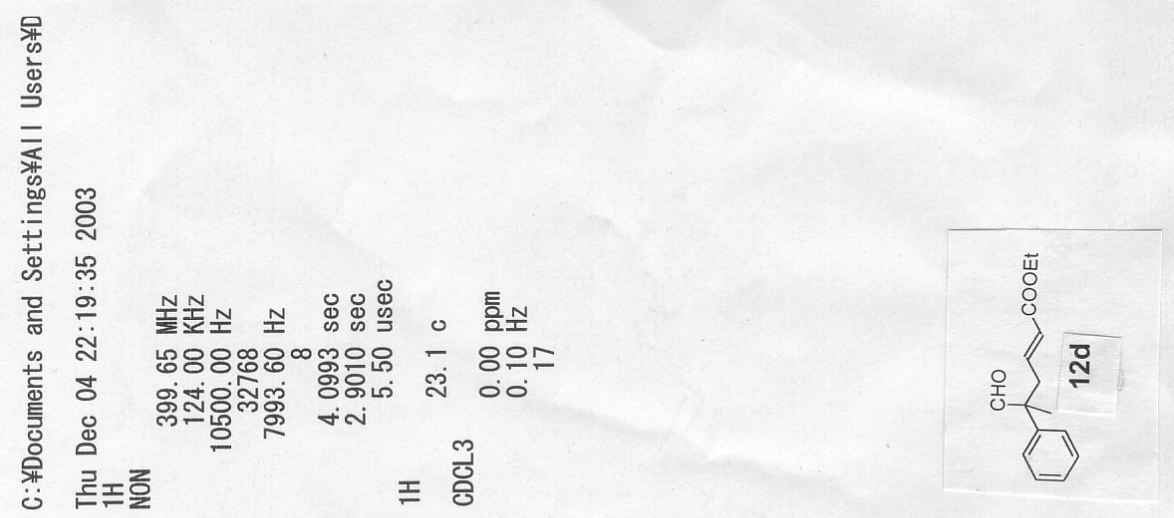

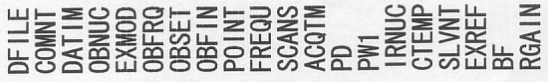

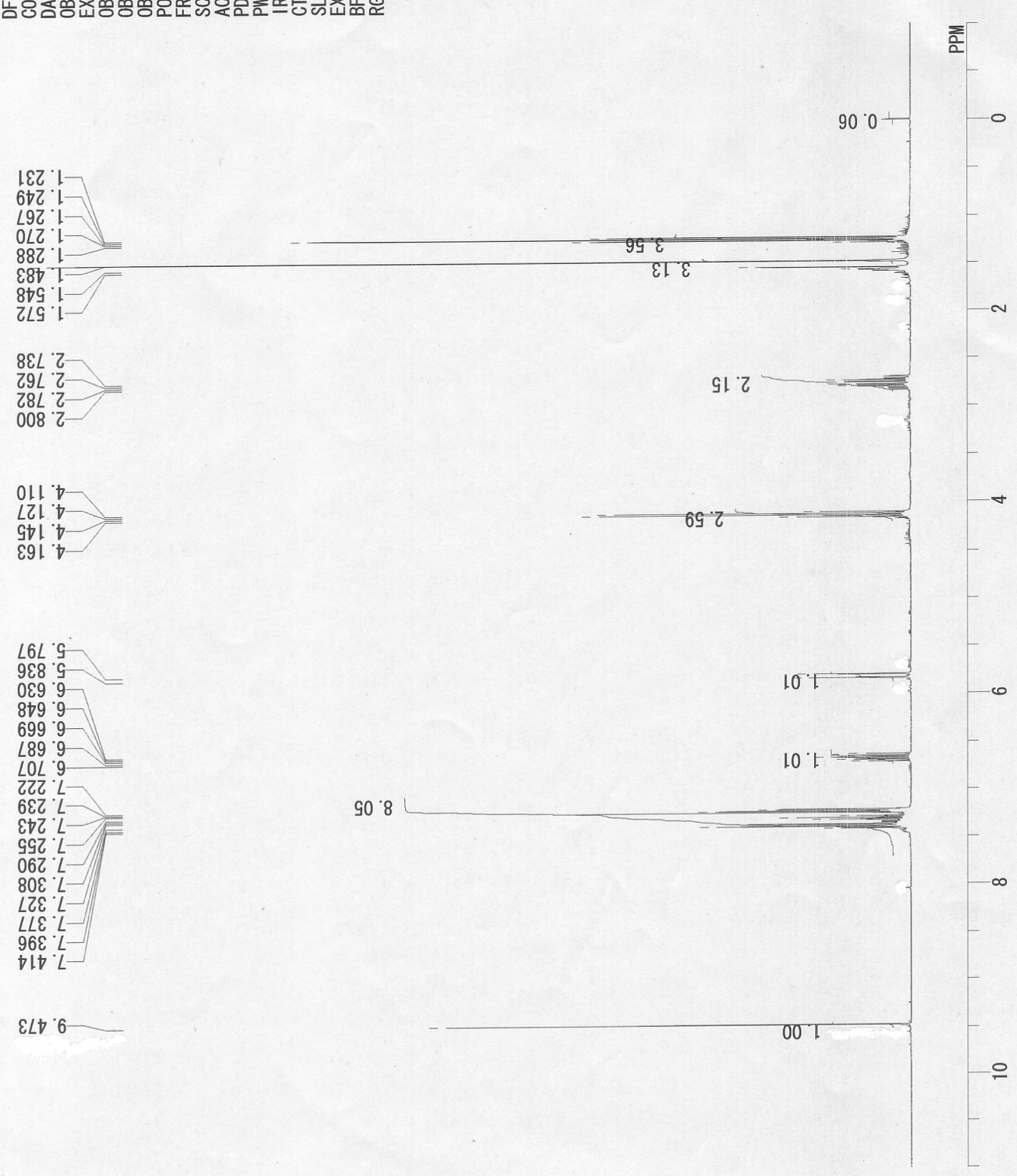



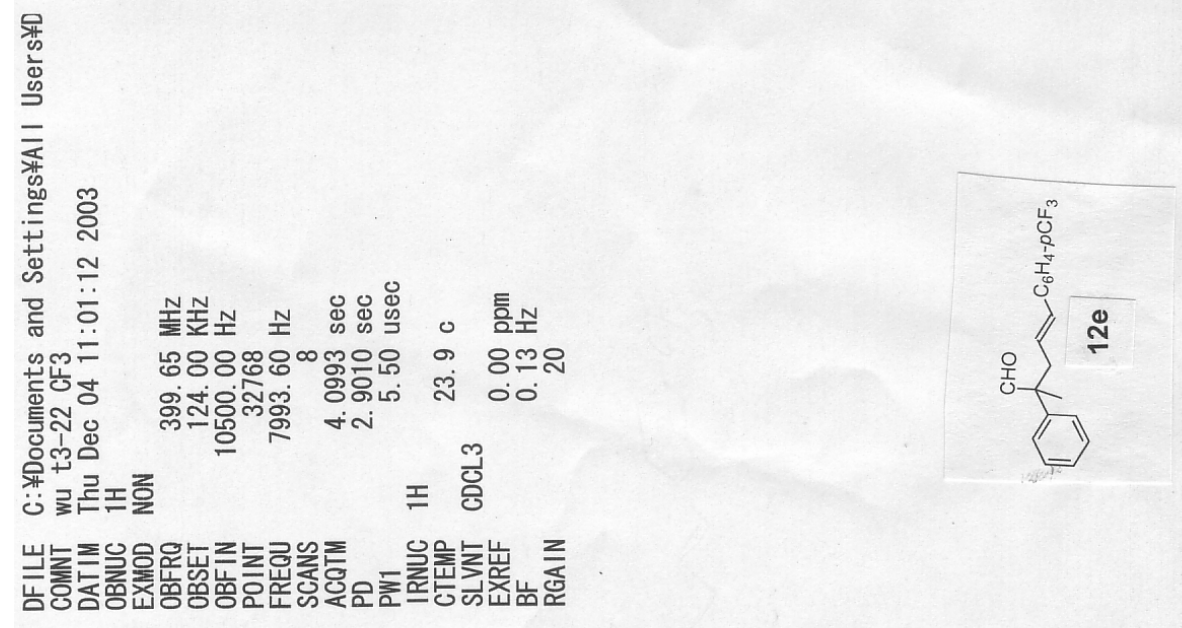

QEt :-

8IS 1

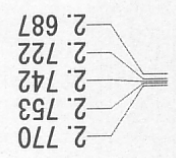

$126^{\circ} 9$

$076{ }^{\circ} 9$

096.9

$866^{\circ} \mathrm{9}$

9 IE 9

GSE 9

1812

$68 \mathrm{I}^{\circ} \mathrm{L}$

$80 Z \cdot L$

$\angle \nabla Z$

092

$81 \varepsilon^{\circ} L$

$8 \mathcal{E} \mathcal{E}^{\circ} \mathrm{L}$

$99 \varepsilon^{\circ} L$

tht L

GEt $L$

$287^{\circ} 6$

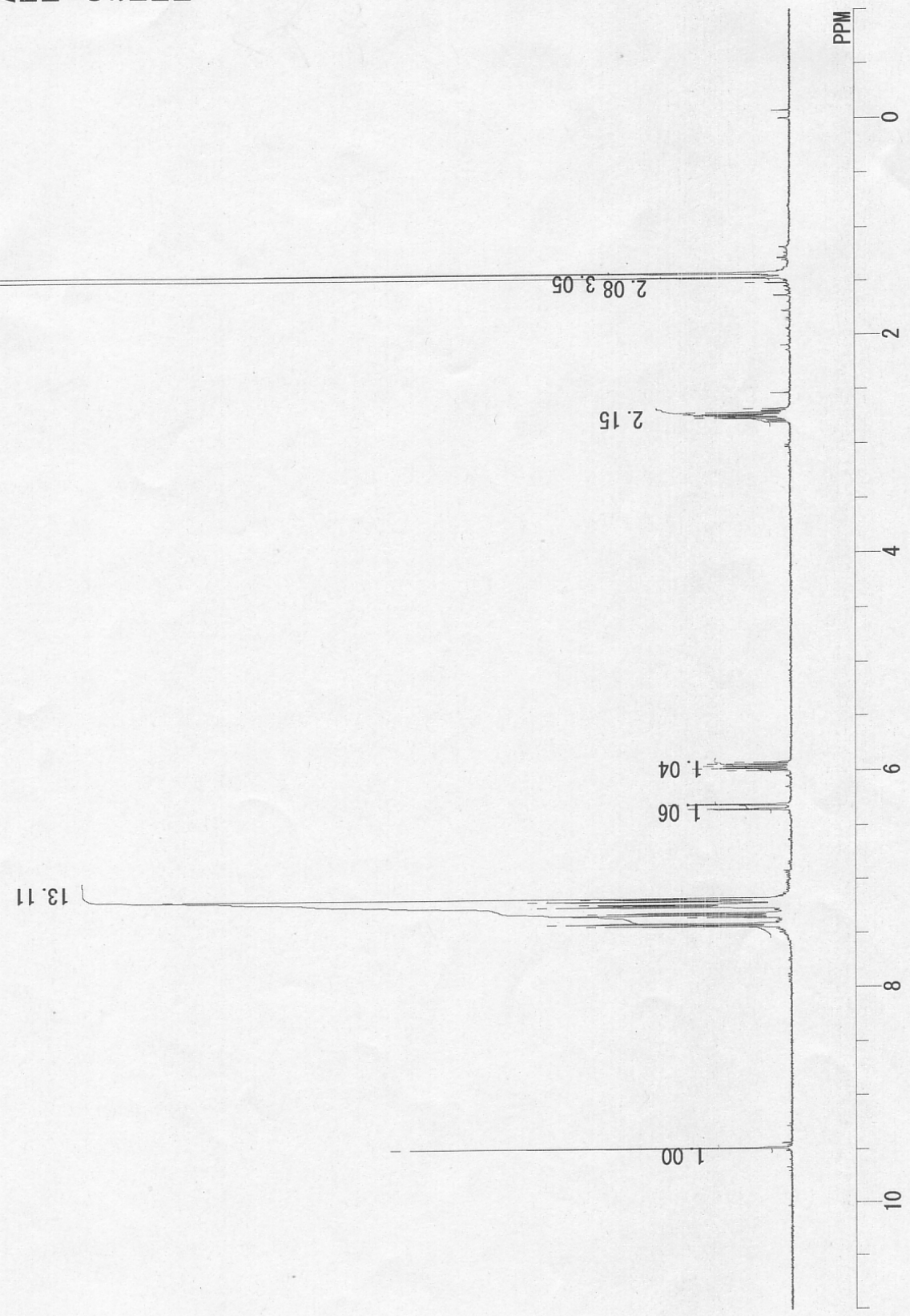



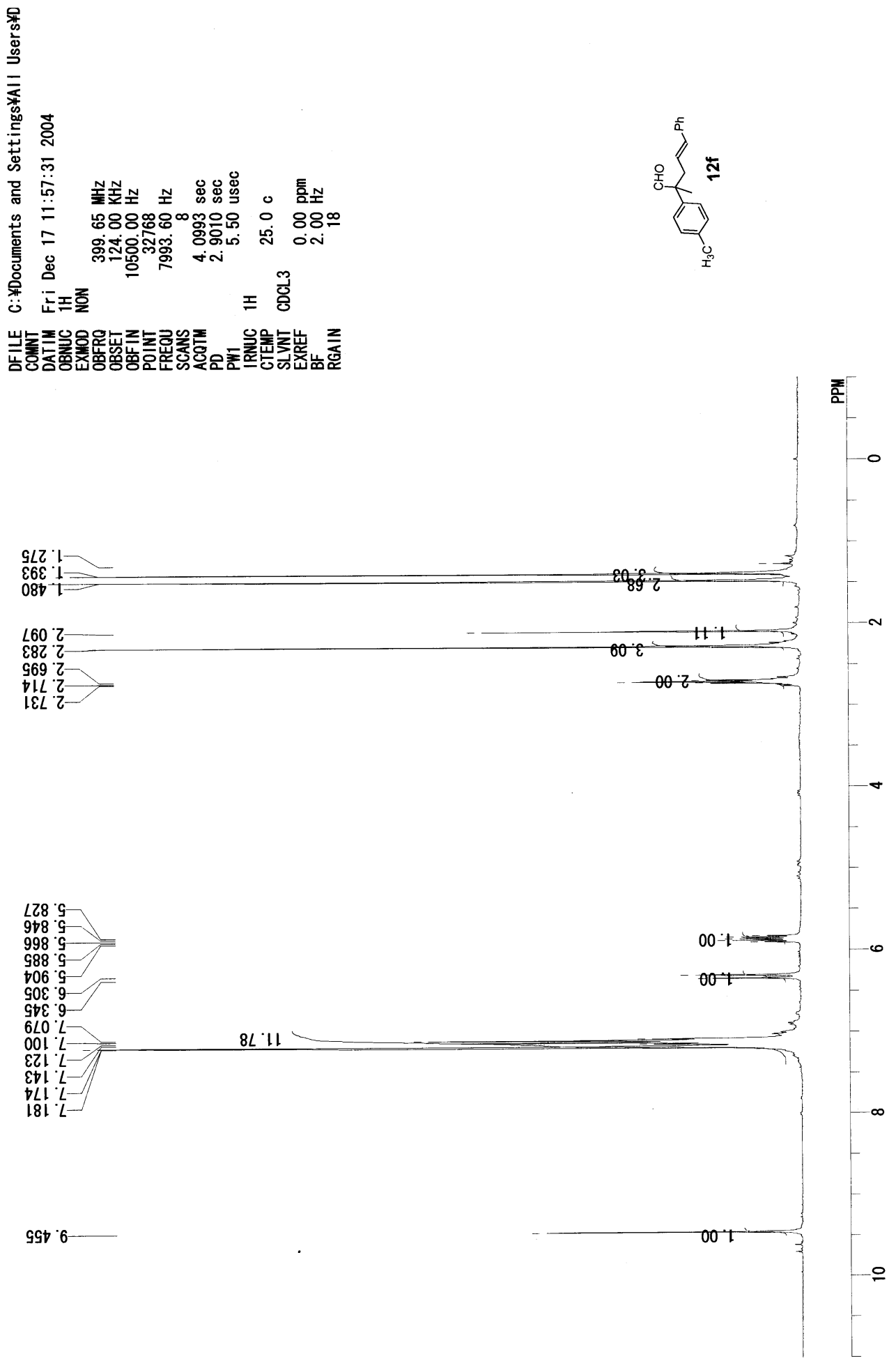

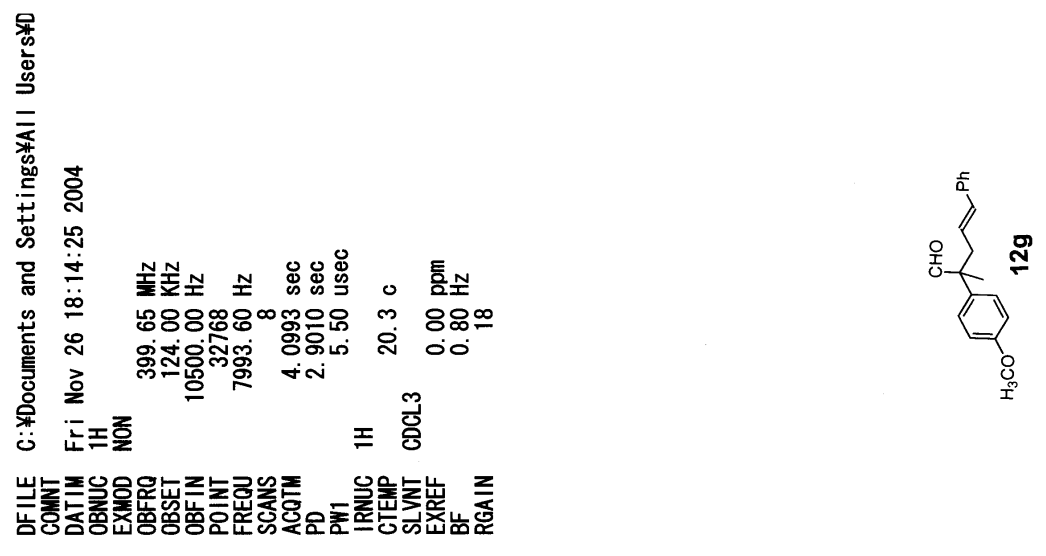

$000 \%$

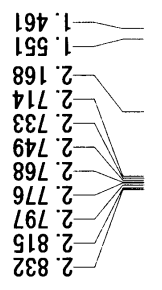

918

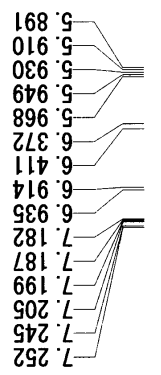

$209^{\circ} 6$
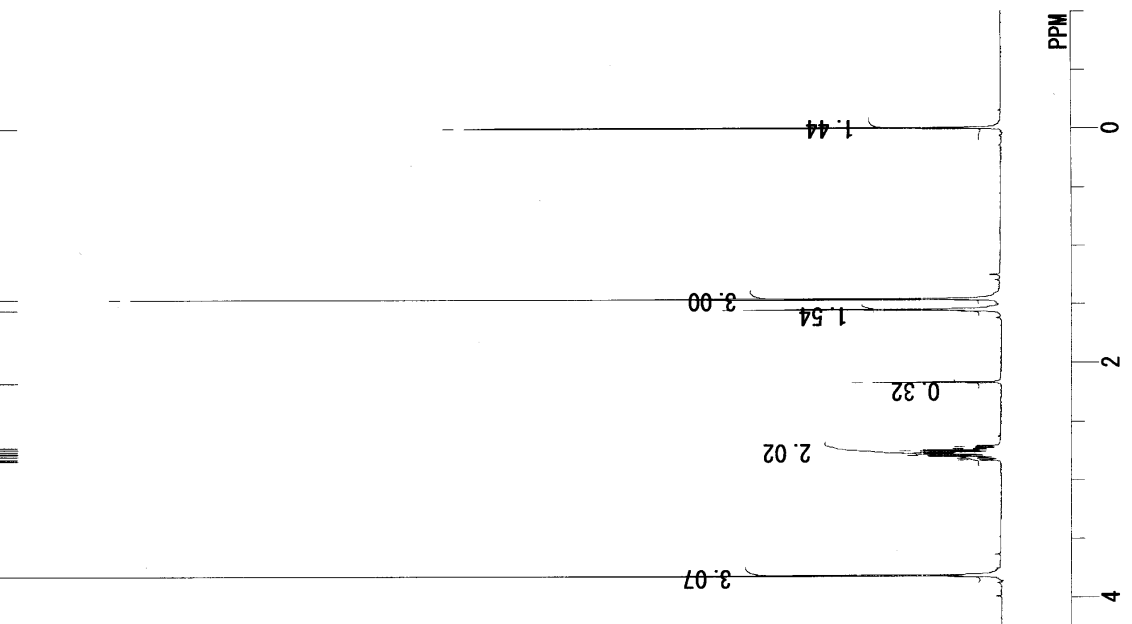

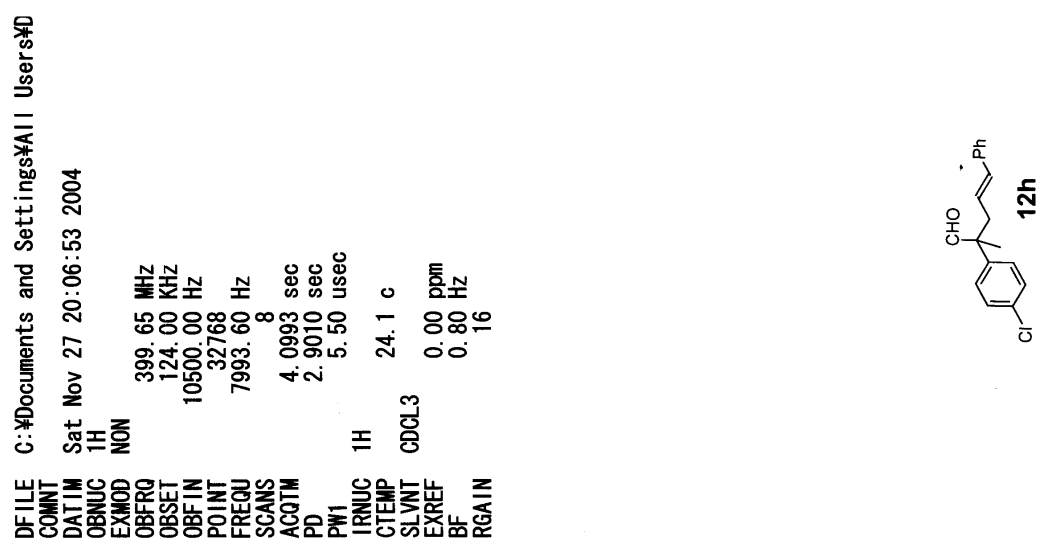

$000^{\circ} 0$

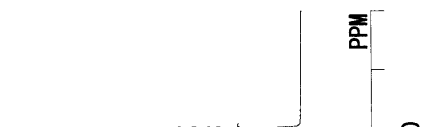

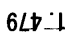

GSS

$\angle 91$
$81 L$

ESL Z

OLL Z

118 2

678
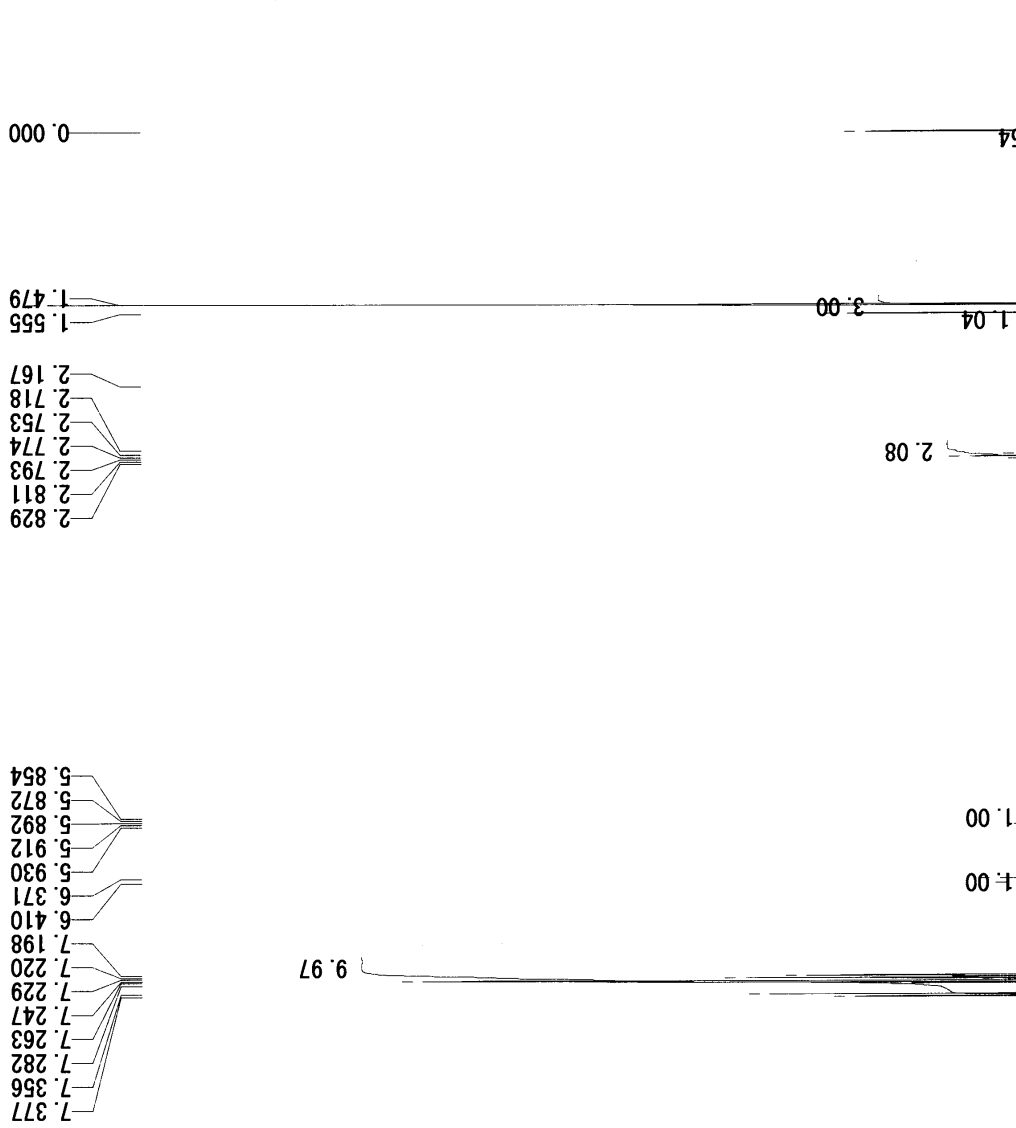

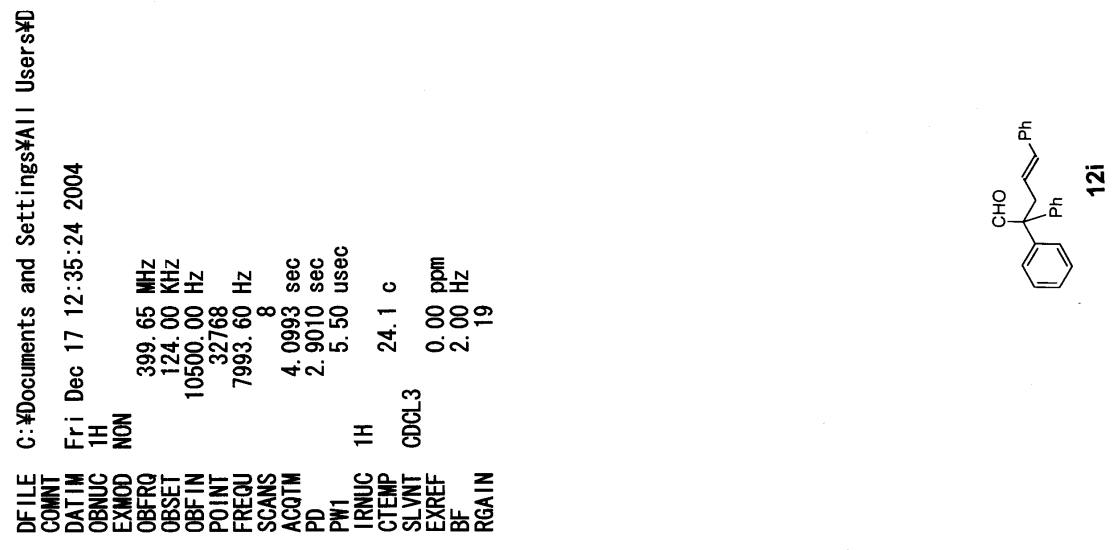

\&૬9 ${ }^{\circ}-$
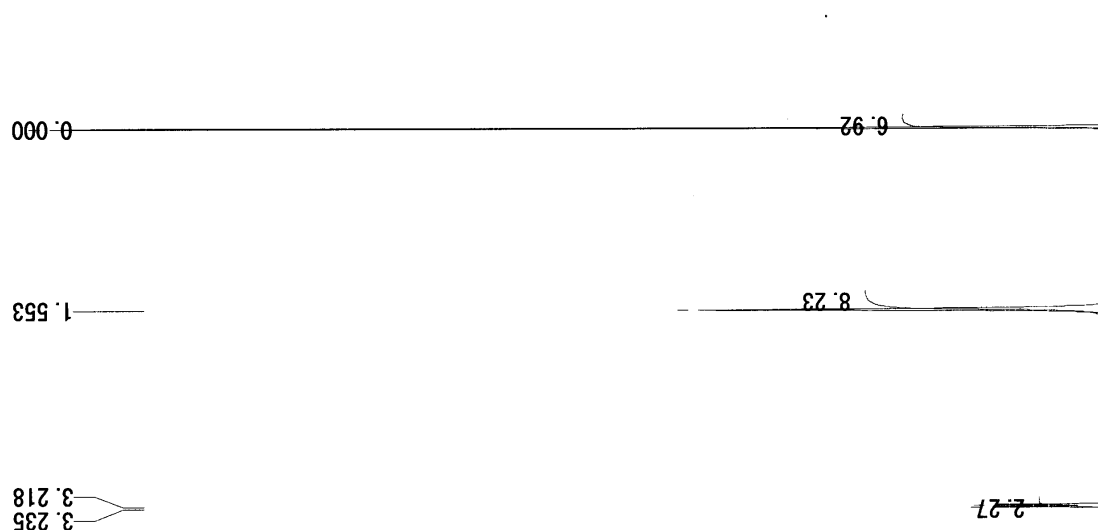

$000 \div 0$

36.9
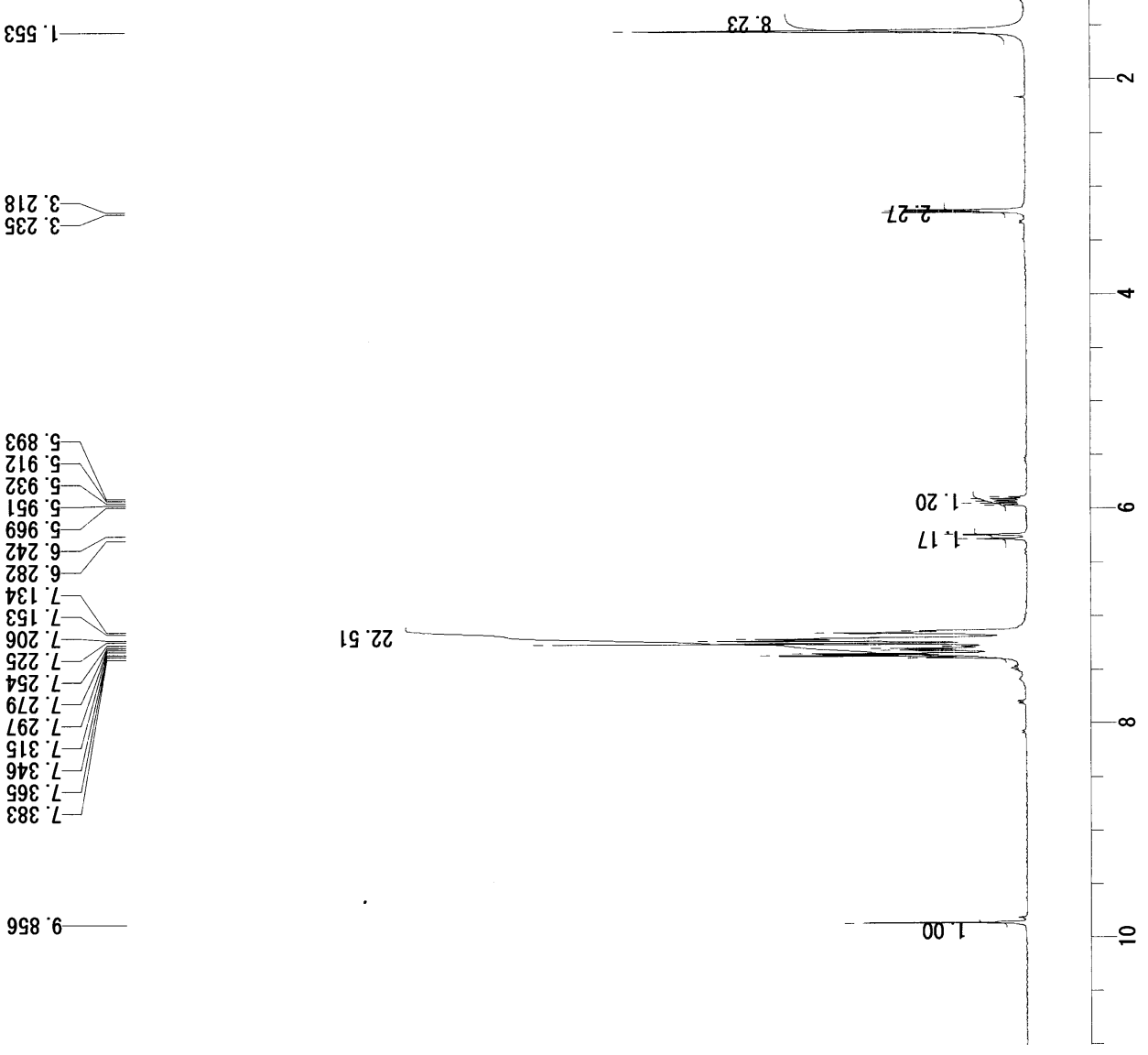

$998^{\circ} 6$ 

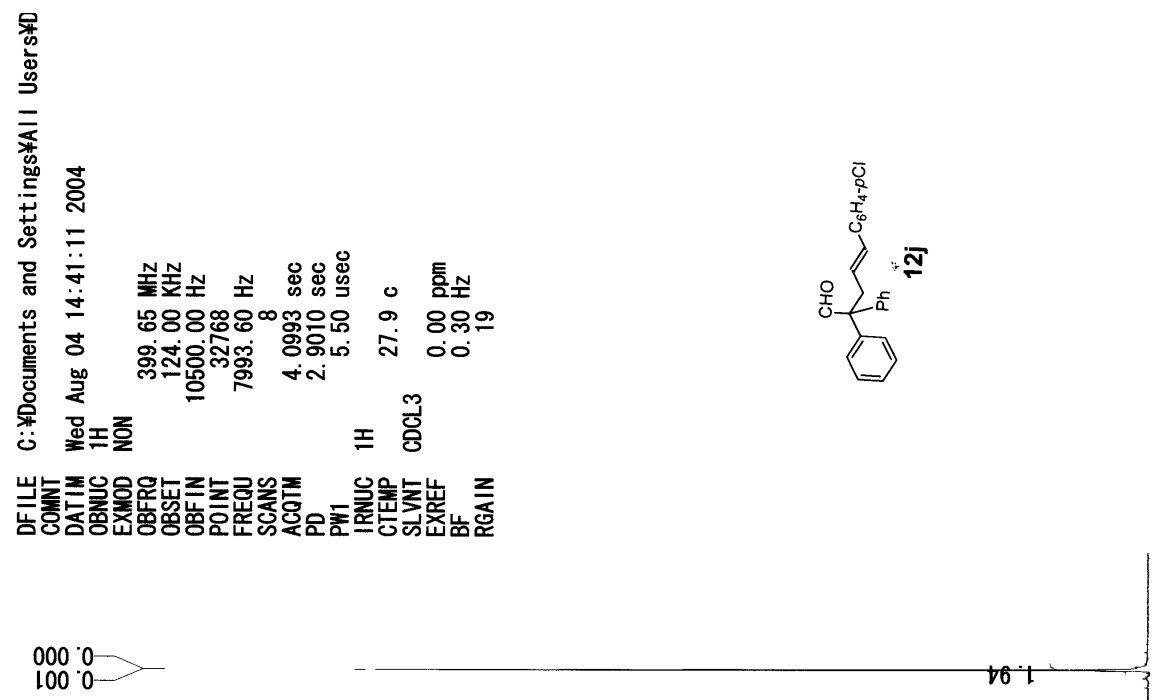

†०S 1
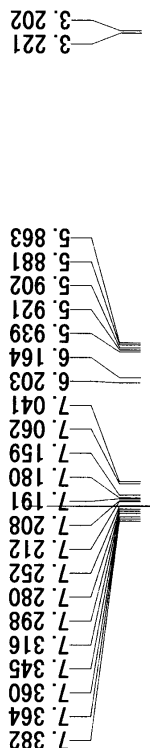

$098 \cdot 6$
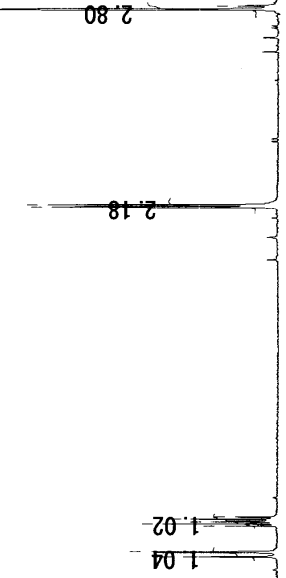

001

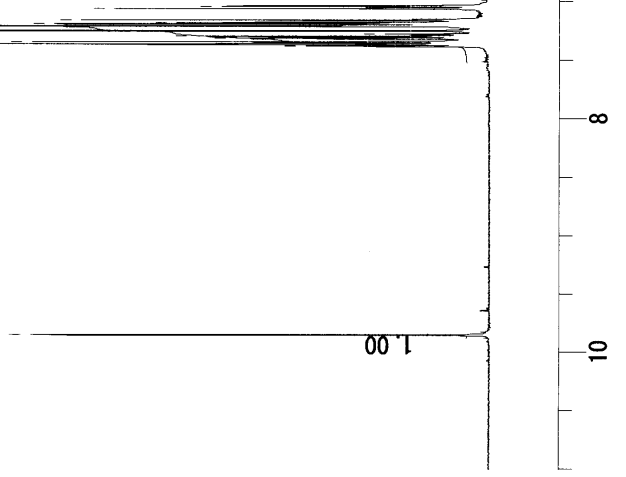

